\title{
On the chiral expansion of vector meson masses
}

\author{
R. Bavontaweepanya, ${ }^{1}$ Xiao-Yu Guo, ${ }^{2}$ and M. F. M. Lutz ${ }^{2,3}$ \\ ${ }^{1}$ Department of Physics, Faculty of Science, Mahidol University, Bangkok 10400, Thailand \\ ${ }^{2}$ GSI Helmholtzzentrum für Schwerionenforschung GmbH, Planckstraße 1, 64291 Darmstadt, Germany \\ ${ }^{3}$ Technische Universität Darmstadt, D-64289 Darmstadt, Germany
}

(Received 31 January 2018; published 10 September 2018)

\begin{abstract}
We study the chiral expansion of meson masses and decay constants using a chiral Lagrangian that was constructed previously based on the hadrogenesis conjecture. The one-loop self-energies of the Goldstone bosons and vector mesons are evaluated. It is illustrated that a order-by-order renormalizable effective field theory arises once specific conditions on the low-energy constants are imposed. For the case where the hadrogenesis mass gap scale $\Lambda_{\mathrm{HG}}$ is substantially larger than the chiral symmetry breaking scale $\Lambda_{\chi}$ a partial summation scheme is required. All terms proportional to $\left(M / \Lambda_{\chi}\right)^{n}$ can be summed by a suitable renormalization, where $M$ is the chiral and large- $N_{c}$ limit of the vector meson masses in QCD. The size of loop effects from vector meson degrees of freedom is illustrated for physical quark masses. Naturally sized effects are observed that have significant impact on the chiral structure of low-energy QCD with three light flavors.
\end{abstract}

DOI: 10.1103/PhysRevD.98.056005

\section{INTRODUCTION}

Vector meson degrees of freedom are known to play an important role in hadron physics. Since the seminal work of Sakurai [1] pioneering the vector meson dominance phenomenology, there is the quest how such a picture can be related to the underlying fundamental theory of strong interactions. To the best knowledge of the authors such a link to QCD has not been established so far [2-14].

There is a rather successful chiral Lagrangian originally constructed by Bando and coauthors, where the vector mesons are considered as non-Abelian gauge bosons properly coupled to the Goldstone bosons in compliance with the chiral Ward identities of QCD [15-18]. Related works that consider in addition explicit scalar and axialvector fields are scrutinized in [19-22]. The challenge of such a path is the question whether the effective Lagrangian is general enough as to guarantee a systematic link to QCD as its low-energy effective field theory. Is there any powercounting principle that generalizes the Lagrangian and permits a consistent renormalization program? An alternative starting point is a chiral Lagrangian where vector meson degrees of freedom are considered as heavy fields initially [23-28]. An infinite tower of interaction terms can readily be written down. However, it is unclear how to order

Published by the American Physical Society under the terms of the Creative Commons Attribution 4.0 International license. Further distribution of this work must maintain attribution to the author(s) and the published article's title, journal citation, and DOI. Funded by SCOAP ${ }^{3}$. this plethora of terms and how to consider the loop effects implied $[11,29]$. In both approaches the challenge is caused by meson resonances that are close to the vector mesons in mass [30-32]. Is there any rationality to construct a chiral Lagrangian with vector mesons but to leave out, for instance, scalar and axial vector mesons? Indeed, the hadrogenesis conjecture proposes such a scenario: meson resonances that are not considered as explicit degrees of freedom in the effective Lagrangian may be dynamically generated by coupled-channel dynamics based on that Lagrangian [33-38]. While for scalar mesons such a mechanism is known since the early days of the quark model $[39,40]$, only a decade ago one of the authors illustrated that chiral symmetry predicts a spectrum of axial-vector mesons as a consequence of the coupledchannel interactions of the Goldstone bosons with vector mesons $[13,35,36,41,42]$. While such results support the hadrogenesis conjecture, it is still an open challenge how to systematize such an approach.

Recently a possible direct link of the hadrogenesis conjecture to QCD was suggested in [14]. If chiral QCD at vanishing up, down, and strange quark masses is considered in the limit of a large number of colors $\left(N_{c}\right)$, an infinite tower of discrete states appears [43]. While it is established that such a tower of states exists, it is not known from first principle where the levels are located. There is no stringent reason that this spectrum resembles closely the excitation spectrum of QCD at finite quark masses and a finite number of colors $N_{c}$. Suppose that there would be a significant mass gap below some hard scale $\Lambda_{\mathrm{HG}}$ in chiral QCD at large $N_{c}$. This would permit the construction of an 
effective field theory description for the physics below that heavy scale $\Lambda_{\mathrm{HG}}$. The relevant degrees of freedom are identified with the states in the spectrum that are below that scale. A possible minimal scenario would be that those relevant degrees of freedom are the Goldstone bosons accompanied by the light vector mesons only. Based on this assumption the leading order chiral Lagrangian was constructed in [14]. The power counting is based on the assumption that $M_{V} / \Lambda_{\mathrm{HG}} \sim Q$ is sufficiently small as to arrive at a convergent expansion.

While in [14] a chiral Lagrangian was constructed according to a dimensional power counting scheme in the presence of a conjectured hadrogenesis scale $\Lambda_{\mathrm{HG}}$, its consistency as an effective field theory remained an open issue. In particular, can it be renormalized convincingly $[44,45]$ ? This question will be studied at the one-loop level in this work. It is well known from various quantum field theories that the quest of renormalizability may impose stringent conditions on the form of the effective Lagrangian. In our approach we use dimensional regularization, where there are no explicit power divergencies. The latter are absorbed into the coupling constant, with the possibility of fine-tuned low-energy parameters. The theory is not renormalizable in the strict sense, but given a power counting it may be renormalized order by order as it is in chiral perturbation theory $(\chi \mathrm{PT})$.

The work is organized as follows. In Sec. II we recall the chiral Lagrangian as constructed in [14]. Additional terms of order $Q^{4}$ are constructed that are required for the renormalization of the one-loop contributions to the meson masses. It follows in Secs. III and IV where the one-loop contributions to the meson masses are computed and analyzed. Explicit results on the scale dependence of the low-energy constants are derived. The importance of explicit vector meson degrees of freedom is illustrated by a series of figures that detail the one-loop contributions to the meson masses. In Sec. V the decay constants of the Goldstone bosons are considered. In Section VI, various scenarios for the typical size of the low-energy constants are presented. Section VII gives a short summary and outlook.

\section{THE CHIRAL LAGRANGIAN WITH LIGHT VECTOR MESON FIELDS}

We recall the hadrogenesis Lagrangian as introduced in [14]. A chiral $S U(3)$ Lagrangian is readily constructed utilizing appropriate building blocks [6,46-50]. The basic elements are

$$
\begin{aligned}
U_{\mu}= & \frac{1}{2} e^{-i \frac{\Phi}{2 f}}\left(\partial_{\mu} e^{i \frac{\Phi}{f}}\right) e^{-i \frac{\Phi}{2 f}}-\frac{i}{2} e^{-i \frac{\Phi}{2 f}} r_{\mu} e^{+i \frac{\Phi}{2 f}} \\
& +\frac{i}{2} e^{+i \frac{\Phi}{2 f}} l_{\mu} e^{-i \frac{\Phi}{2 f}}, \quad \Phi_{\mu \nu}, \quad H=\frac{1}{\sqrt{6} f} \operatorname{tr} \Phi, \\
f_{\mu \nu}^{ \pm}= & \frac{1}{2} e^{+i \frac{\Phi}{2 f}}\left(\partial_{\mu} l_{\nu}-\partial_{\nu} l_{\nu}-i\left[l_{\mu}, l_{\nu}\right]_{-}\right) e^{-i \frac{\Phi}{2 f}} \\
& \pm \frac{1}{2} e^{-i \frac{\Phi}{2 f}}\left(\partial_{\mu} r_{\nu}-\partial_{\nu} r_{\nu}-i\left[r_{\mu}, r_{\nu}\right]_{-}\right) e^{+i \frac{\Phi}{2 f}},
\end{aligned}
$$

where we include a nonet of pseudoscalar-meson fields $\Phi\left(J^{P}=0^{-}\right)$and a nonet of vector meson fields in the antisymmetric tensor representation $\Phi_{\mu \nu}\left(J^{P}=1^{-}\right)$. The notations and conventions of [14] are used throughout this work. The classical source functions $r_{\mu}$ and $l_{\mu}$ in (1) are linear combinations of the vector and axial-vector sources of QCD with $r_{\mu}=v_{\mu}+a_{\mu}$ and $l_{\mu}=v_{\mu}-a_{\mu}$. Explicit chiral symmetry-breaking effects are included in terms of scalar and pseudoscalar source fields $\chi_{ \pm}$proportional to the quark-mass matrix of QCD,

$$
\chi_{ \pm}=\frac{1}{2}\left(e^{+i \frac{\Phi}{2 f}} \chi_{0} e^{+i \frac{\Phi}{2 f}} \pm e^{-i \frac{\Phi}{2 f}} \chi_{0} e^{-i \frac{\Phi}{2 f}}\right),
$$

where $\chi_{0}=2 B_{0} \operatorname{diag}\left(m_{u}, m_{d}, m_{s}\right)$.

The covariant derivative $D_{\mu}$ discriminates flavor octet from flavor singlet fields. It is identical for all matrix fields in (1) and (2),

$$
\begin{aligned}
D_{\mu} O= & \partial_{\mu} O+\left[\Gamma_{\mu}, O\right]_{-}, \quad D_{\mu} H=\partial_{\mu} H-\sqrt{\frac{2}{3}} \operatorname{tr}\left(a_{\mu}\right), \\
\Gamma_{\mu}= & \frac{1}{2} e^{-i \frac{\Phi}{2 f}}\left[\partial_{\mu}-i\left(v_{\mu}+a_{\mu}\right)\right] e^{+i \frac{\Phi}{2 f}} \\
& +\frac{1}{2} e^{+i \frac{\Phi}{2 f}}\left[\partial_{\mu}-i\left(v_{\mu}-a_{\mu}\right)\right] e^{-i \frac{\Phi}{2 f}}
\end{aligned}
$$

with $O \in\left\{U_{\mu}, \Phi_{\mu \nu}, f_{\mu \nu}^{ \pm}, \chi_{ \pm}\right\}$and the chiral connection $\Gamma_{\mu}$. In a covariant derivative on the singlet field $H$ the axial source function $a_{\mu}$ is probed only.

In the following we focus on terms established previously in $[13,14]$ that do not involve the flavor singlet field $H$. At second order the various terms can be grouped into three classes:

$$
\begin{aligned}
& \mathcal{L}_{2}^{(2)}=-\frac{1}{4} \operatorname{tr}\left\{\left(D^{\mu} \Phi_{\mu \alpha}\right)\left(D_{\nu} \Phi^{\nu \alpha}\right)\right\}+\frac{1}{8} M^{2} \operatorname{tr}\left\{\Phi^{\mu \nu} \Phi_{\mu \nu}\right\}+\frac{1}{2} f_{V} \operatorname{tr}\left\{\Phi^{\mu \nu} f_{\mu \nu}^{+}\right\}-f^{2} \operatorname{tr}\left\{U_{\mu} U^{\mu}\right\}+\frac{1}{2} f^{2} \operatorname{tr}\left\{\chi_{+}\right\}, \\
& \mathcal{L}_{2}^{(3)}=\frac{i}{2} f h_{1} \operatorname{tr}\left\{U_{\mu} \Phi^{\mu \nu} U_{\nu}\right\}+\frac{i}{8} h_{2} \varepsilon^{\mu \nu \alpha \beta} \operatorname{tr}\left\{\left[\Phi_{\mu \nu},\left(D^{\tau} \Phi_{\tau \alpha}\right)\right]_{+} U_{\beta}\right\}-\frac{i}{4} \frac{M^{2}}{f} h_{3} \operatorname{tr}\left\{\Phi_{\mu \tau} \Phi^{\mu \nu} \Phi_{\nu}^{\tau}\right\},
\end{aligned}
$$




$$
\begin{aligned}
\mathcal{L}_{2}^{(4)}= & \frac{1}{8} g_{1} \operatorname{tr}\left\{\left[\Phi_{\mu \nu}, U_{\alpha}\right]_{+}\left[U^{\alpha}, \Phi^{\mu \nu}\right]_{+}\right\}+\frac{1}{8} g_{2} \operatorname{tr}\left\{\left[\Phi_{\mu \nu}, U_{\alpha}\right]_{-}\left[U^{\alpha}, \Phi^{\mu \nu}\right]_{-}\right\}+\frac{1}{8} g_{3} \operatorname{tr}\left\{\left[U_{\mu}, U^{\nu}\right]_{+}\left[\Phi_{\nu \tau}, \Phi^{\mu \tau}\right]_{+}\right\} \\
& +\frac{1}{8} g_{4} \operatorname{tr}\left\{\left[U_{\mu}, U^{\nu}\right]_{-}\left[\Phi_{\nu \tau}, \Phi^{\mu \tau}\right]_{-}\right\}+\frac{1}{8} g_{5} \operatorname{tr}\left\{\left[\Phi^{\mu \tau}, U_{\mu}\right]_{-}\left[\Phi_{\nu \tau}, U^{\nu}\right]_{-}\right\}+\frac{1}{8} \frac{M^{2}}{f^{2}} g_{6} \operatorname{tr}\left\{\left[\Phi_{\mu \nu}, \Phi_{\alpha \beta}\right]_{+}\left[\Phi^{\alpha \beta}, \Phi^{\mu \nu}\right]_{+}\right\} \\
& +\frac{1}{8} \frac{M^{2}}{f^{2}} g_{7} \operatorname{tr}\left\{\left[\Phi_{\alpha \beta}, \Phi_{\mu \nu}\right]_{-}\left[\Phi^{\alpha \beta}, \Phi^{\mu \nu}\right]_{-}\right\}+\frac{1}{8} \frac{M^{2}}{f^{2}} g_{8} \operatorname{tr}\left\{\left[\Phi^{\mu \nu}, \Phi_{\mu \beta}\right]_{+}\left[\Phi_{\alpha \nu}, \Phi^{\alpha \beta}\right]_{+}\right\}+\frac{1}{8} \frac{M^{2}}{f^{2}} g_{9} \operatorname{tr}\left\{\left[\Phi^{\mu \nu}, \Phi_{\mu \beta}\right]_{-}\left[\Phi_{\alpha \nu}, \Phi^{\alpha \beta}\right]_{-}\right\},
\end{aligned}
$$

where we recall the counting scheme with $D_{\mu}, U_{\mu} \sim Q$ but $\chi_{ \pm} \sim Q^{2}$. The scale $M \sim Q$ is counted as order one, if it is probed relative to the hadrogenesis scale $\Lambda_{\mathrm{HG}}$ with $M / \Lambda_{\mathrm{HG}} \sim Q$ (see [14]).

It is well known from various quantum field theories that the quest of renormalizability may impose stringent conditions on the form of the effective Lagrangian. Indeed, we already omitted three terms initially suggested in [14] to enter the Lagrangian at order $Q^{2}$. We anticipate the outcome of our study, which requires that these three terms contribute at order $Q^{4}$ only. The first term,

$$
\frac{1}{8} b_{D} \operatorname{tr}\left\{\Phi^{\mu \nu} \Phi_{\mu \nu} \chi_{+}\right\} \rightarrow \frac{1}{8} b_{1} M^{2} \operatorname{tr}\left\{\Phi^{\mu \nu} \Phi_{\mu \nu} \chi_{+}\right\},
$$

breaks chiral symmetry explicitly. By assigning to its structure the factor $M^{2}$ it is moved from $\mathcal{L}_{2}$ to $\mathcal{L}_{4}$. This implies that all vector meson masses are given by $M$ at leading order in our counting scheme. Without such a property we do not see any path for a consistent renormalization program. The case for the other two terms,

$$
\frac{i}{8} h_{4} \varepsilon^{\mu \nu \alpha \beta} \operatorname{tr}\left\{\left[\left(D_{\alpha} \Phi_{\mu \nu}\right), \Phi_{\tau \beta}\right]_{+} U^{\tau}\right\}, \quad \frac{i}{4} h_{5} \varepsilon^{\mu \nu \alpha \beta} \operatorname{tr}\left\{\Phi_{\mu \nu} \chi_{-} \Phi_{\alpha \beta}\right\},
$$

is more intricate. If considered at order $Q^{2}$ they would generate a scale dependence at the one-loop level that cannot be absorbed into the available counterterms at order $Q^{4}$. Therefore we insist on $h_{4,5} \rightarrow M^{2} h_{4,5}$ as well. Thus such terms contribute to $\mathcal{L}_{4}$ and therefore turn irrelevant for the one-loop study of this work.

Some of the low-energy parameters have been estimated before in $[13,14]$ with

$$
\begin{aligned}
h_{1}=f_{V} h_{P} / f & \simeq 2.5 \pm 0.25, \quad h_{2}=h_{A}=2.33 \pm 0.03, \\
h_{3} & =f h_{V} / f_{V} \simeq 0.05,
\end{aligned}
$$

where $h_{P}, h_{A}, h_{V}$ are the low-energy constants as introduced in [14]. The parameter $f \simeq 90 \mathrm{MeV}$ is the chiral limit value of the pion or kaon decay constant. The tree-level estimate for the parameter $b_{1}$ from [14] should be rejected since according to our findings it should be determined in the presence of one-loop effects. For the remaining constants $g_{i}$ so far no reliable estimate exists.

Since we will compute the one-loop contributions to the Goldstone boson and vector meson self-energies, we need to collect an appropriate set of counterterms to renormalize their scale dependent parts. According to our power counting the latter are expected to be of order four. While the Goldstone boson sector [51] is well established,

$$
\begin{aligned}
\mathcal{L}_{4}^{(P)}= & 16 L_{1}\left(\operatorname{tr}\left\{U_{\mu} U^{\mu}\right\}\right)^{2}+16 L_{2} \operatorname{tr}\left\{U_{\mu} U_{\nu}\right\} \operatorname{tr}\left\{U^{\mu} U^{\nu}\right\} \\
& +16 L_{3} \operatorname{tr}\left\{U_{\mu} U^{\mu} U_{\nu} U^{\nu}\right\}-8 L_{4} \operatorname{tr}\left\{U_{\mu} U^{\mu}\right\} \operatorname{tr}\left\{\chi_{+}\right\} \\
& -8 L_{5} \operatorname{tr}\left\{U_{\mu} U^{\mu} \chi_{+}\right\}+4 L_{6} \operatorname{tr}\left\{\chi_{+}\right\} \operatorname{tr}\left\{\chi_{+}\right\} \\
& +4 L_{7} \operatorname{tr}\left\{\chi_{-}\right\} \operatorname{tr}\left\{\chi_{-}\right\}+2 L_{8} \operatorname{tr}\left\{\chi_{+} \chi_{+}+\chi_{-} \chi_{-}\right\},
\end{aligned}
$$

this is not the case for the terms involving the light vector mesons. A complete construction of the complete fourth order Lagrangian in the presence of vector meson fields is beyond the scope of our work. Here we focus on the terms that involve two vector meson fields and at most two $\chi_{+}$fields. Altogether we have

$$
\begin{aligned}
\mathcal{L}_{4}^{(V)}= & \frac{e_{1}}{8} M^{4} \operatorname{tr}\left\{\Phi^{\mu \nu} \Phi_{\mu \nu}\right\}+\frac{e_{2}}{8} M^{4} \operatorname{tr}\left\{\Phi_{\mu \nu}\right\} \operatorname{tr}\left\{\Phi^{\mu \nu}\right\} \\
& +\frac{b_{1}}{8} M^{2} \operatorname{tr}\left\{\Phi^{\mu \nu} \Phi_{\mu \nu} \chi_{+}\right\}+\frac{b_{2}}{8} M^{2} \operatorname{tr}\left\{\Phi_{\mu \nu} \Phi^{\mu \nu}\right\} \operatorname{tr}\left\{\chi_{+}\right\} \\
& +\frac{b_{3}}{8} M^{2} \operatorname{tr}\left\{\Phi_{\mu \nu}\right\} \operatorname{tr}\left\{\Phi^{\mu \nu} \chi_{+}\right\}+\frac{c_{1}}{8} \operatorname{tr}\left\{\Phi_{\mu \nu} \chi_{+} \Phi^{\mu \nu} \chi_{+}\right\} \\
& +\frac{c_{2}}{8} \operatorname{tr}\left\{\Phi_{\mu \nu} \Phi^{\mu \nu} \chi_{+}^{2}\right\},
\end{aligned}
$$

where we do not consider corresponding terms with further number of traces at this order. Any term that involves additional flavor traces is suppressed by the factor $1 / N_{c}$ at least. In our scheme this is translated into the factor $M^{2}$ such as to transport this suppression factor into the dimensional counting rule. We further illustrate our construction principle by a partial list of $Q^{6}$ and $Q^{8}$ terms: 


$$
\begin{aligned}
\mathcal{L}_{6}^{(V)}= & \frac{c_{3}}{8} M^{2} \operatorname{tr}\left\{\Phi_{\mu \nu} \Phi^{\mu \nu}\right\} \operatorname{tr}\left\{\chi_{+}^{2}\right\}+\frac{c_{4}}{8} M^{2} \operatorname{tr}\left\{\Phi_{\mu \nu} \Phi^{\mu \nu} \chi_{+}\right\} \operatorname{tr}\left\{\chi_{+}\right\}+\frac{c_{5}}{8} M^{2} \operatorname{tr}\left\{\Phi^{\mu \nu} \chi_{+}\right\} \operatorname{tr}\left\{\Phi_{\mu \nu} \chi_{+}\right\}+\frac{c_{6}}{8} M^{2} \operatorname{tr}\left\{\Phi^{\mu \nu}\right\} \operatorname{tr}\left\{\Phi_{\mu \nu} \chi_{+}^{2}\right\} \\
& +\frac{b_{4}}{8} M^{4} \operatorname{tr}\left\{\Phi_{\mu \nu}\right\} \operatorname{tr}\left\{\Phi^{\mu \nu}\right\} \operatorname{tr}\left\{\chi_{+}\right\}, \\
\mathcal{L}_{8}^{(V)}= & \frac{c_{7}}{8} M^{4} \operatorname{tr}\left\{\Phi^{\mu \nu} \Phi_{\mu \nu}\right\} \operatorname{tr}\left\{\chi_{+}\right\} \operatorname{tr}\left\{\chi_{+}\right\}+\frac{c_{8}}{8} M^{4} \operatorname{tr}\left\{\Phi^{\mu \nu}\right\} \operatorname{tr}\left\{\Phi_{\mu \nu} \chi_{+}\right\} \operatorname{tr}\left\{\chi_{+}\right\}+\frac{c_{9}}{8} M^{4} \operatorname{tr}\left\{\Phi^{\mu \nu}\right\} \operatorname{tr}\left\{\Phi_{\mu \nu}\right\} \operatorname{tr}\left\{\chi_{+}^{2}\right\} .
\end{aligned}
$$

Note that a further term with four traces is redundant as it can be generated by a suitable combination of terms presented in (11) and (12).

For none of the dimension full parameters $c_{i}$ is a numerical estimate available. According to the hadrogenesis conjecture we expect, for instance, $c_{1,2}^{\text {ren }} \sim \Lambda_{\mathrm{HG}}^{-2}$ with $\Lambda_{\mathrm{HG}}>$ $2 \mathrm{GeV}$ for suitably renormalized low-energy parameters.

We close this section with a comment on the tensor field representation as applied for the vector mesons. In this approach there are three $J^{P}=1^{-}$propagating modes together with three nonpropagating (frozen) $J^{P}=1^{+}$modes at leading order. This will change at the loop level, where we also expect propagating $1^{+}$modes that are generated by coupled-channel dynamics in line with [35]. Such modes may or may not be seen in the dressed vector meson propagator. Whether they are or are not is not relevant since in any case the physical mode is unambiguously obtained from the Goldstone boson vector meson scattering amplitude properly projected onto its partial-wave with $1^{+}$ quantum numbers. The quantitative details of such $1^{+}$ modes should then be dependent on the low-energy constants of the effective Lagrangian, and there is no fundamental reason to keep the $1^{+}$modes in the vector meson propagator frozen.

\section{VECTOR MESON MASSES AT THE ONE-LOOP LEVEL}

We begin with a collection of all tree-level expressions for the vector meson masses from $\mathcal{L}_{4}^{(V)}$ in (11) for which we find the result

$$
\begin{aligned}
\Pi_{\rho}^{\text {tree }}= & e_{1} M^{4}+2 b_{1} M^{2} B_{0} m+2 b_{2} M^{2} B_{0}\left(2 m+m_{s}\right) \\
& +4\left(c_{1}+c_{2}\right) B_{0}^{2} m^{2}, \\
\Pi_{\omega}^{\text {tree }}= & e_{1} M^{4}+2 e_{2} M^{4}+2\left(b_{1}+2 b_{3}\right) M^{2} B_{0} m \\
& +2 b_{2} M^{2} B_{0}\left(2 m+m_{s}\right)+4\left(c_{1}+c_{2}\right) B_{0}^{2} m^{2}, \\
\Pi_{K^{*}}^{\text {tree }}= & e_{1} M^{4}+b_{1} M^{2} B_{0}\left(m+m_{s}\right)+2 b_{2} M^{2} B_{0}\left(2 m+m_{s}\right) \\
& +4\left(c_{1}+c_{2}\right) B_{0}^{2} m m_{s}+2 c_{2} B_{0}^{2}\left(m-m_{s}\right)^{2}, \\
\Pi_{\phi}^{\text {tree }}= & \left(e_{1}+e_{2}\right) M^{4}+2\left(b_{1}+b_{3}\right) M^{2} B_{0} m_{s} \\
& +2 b_{2} M^{2} B_{0}\left(2 m+m_{s}\right)+4\left(c_{1}+c_{2}\right) B_{0}^{2} m_{s}^{2}, \\
\Pi_{\omega \phi}^{\text {tree }}= & \sqrt{2} e_{2} M^{4}+\sqrt{2} b_{3} M^{2} B_{0}\left(m+m_{s}\right),
\end{aligned}
$$

where a projection of the polarization tensor on its mass component is understood. One may introduce an $\omega-\phi$ mixing angle $\epsilon$ by [52,53]

$$
\omega=\omega^{\prime} \cos \epsilon+\phi^{\prime} \sin \epsilon, \quad \phi=\phi^{\prime} \cos \epsilon-\omega^{\prime} \sin \epsilon,
$$

with $\Pi_{\omega \phi}=\frac{1}{2}\left(\Pi_{\phi}-\Pi_{\omega}\right) \tan (2 \epsilon)$.

In (13) we use a convention where the $\omega$ field has no strangeness content. In the transformed field $\omega^{\prime}$ the mixing angle $\epsilon$ is a direct measure for the latter.

While at order $Q^{2}$ the mass term contribution proportional to $M^{2}$ from $\mathcal{L}_{2}^{(2)}$ in (4) does not predict a mixing of the $\omega$ and $\phi$ mesons, and this is no longer true once the counterterms relevant at $Q^{4}$ are considered. The leading mixing effect is induced by the parameters $e_{2}$ and $b_{3}$. The leading terms proportional to the square of a quark mass, $c_{1}$ and $c_{2}$, do not induce mixing effects [54].

We continue with a coherent documentation of the oneloop contributions to the vector meson self-energies, which are decomposed into a tadpole and a bubble contribution with

$$
\Pi_{V}^{\text {loop }}=\Pi_{V}^{\text {tadpole }}+\Pi_{V}^{\text {bubble }} .
$$

It is convenient to start with terms that result from two-body vertices involving two vector meson fields in (4) and (6). Tadpole structures arise where either a Goldstone boson tadpole $\bar{I}_{Q}$ with $Q \in[8]$ or a vector meson tadpole $\bar{I}_{V}$ with $V \in[9]$ is formed. We express our result

$$
\begin{aligned}
\Pi_{V \in[9]}^{\mathrm{tadpole}}= & \frac{1}{4 f^{2}} \sum_{Q \in[8]}\left\{-2 m_{Q}^{2}\left(4 G_{V Q}^{(S)}+G_{V Q}^{(T)}\right) \bar{I}_{Q}-4 G_{V Q}^{(T)} \bar{I}_{Q}^{(2)}\right\} \\
& +\frac{3}{4 f^{2}} \sum_{R \in[9]} 128 G_{V R}^{(T)} M^{2} \bar{I}_{R}
\end{aligned}
$$

with the tadpole function

$$
\bar{I}_{Q}=\frac{m_{Q}^{2}}{(4 \pi)^{2}} \log \frac{m_{Q}^{2}}{\mu^{2}}, \quad \bar{I}_{Q}^{(2)}=\frac{1}{4} m_{Q}^{2} \bar{I}_{Q},
$$

recalled in its infinite volume limit. The vector meson tadpole $\bar{I}_{R}$ follows from $\bar{I}_{Q}$ with the replacement $m_{Q} \rightarrow M_{R}$. Our derivation of (16) is valid in a finite box, where different 
TABLE I. The coupling constants $G_{V Q}^{(S)}, G_{V Q}^{(T)}$ and $G_{V R}^{(T)}$ as introduced in (16).

\begin{tabular}{|c|c|c|c|c|c|}
\hline V & $Q$ & $G_{V Q}^{(S)}$ & $\begin{array}{l}G_{V Q}^{(T)} \\
\end{array}$ & $R$ & $G_{V R}^{(T)}$ \\
\hline$\rho \rho$ & $\begin{array}{c}\pi \\
K \\
\eta\end{array}$ & $\begin{array}{c}\frac{1}{2} g_{1}+g_{2} \\
\frac{1}{2}\left(g_{1}+g_{2}\right) \\
\frac{1}{6} g_{1}\end{array}$ & $\begin{array}{c}g_{3}-\frac{2}{3} g_{5} \\
\frac{1}{3}\left(2 g_{3}-g_{5}\right) \\
\frac{1}{9} g_{3}\end{array}$ & $\begin{array}{c}\rho \\
K^{*} \\
\omega \\
\phi\end{array}$ & $\begin{array}{c}-\frac{5}{6}\left(g_{6}-g_{7}\right)-\frac{13}{24} g_{8}+\frac{1}{6} g_{9} \\
-\frac{3}{4} g_{6}+\frac{5}{12}\left(g_{7}-g_{8}\right)+\frac{1}{12} g_{9} \\
-\frac{2}{3} g_{6}-\frac{7}{24} g_{8} \\
0\end{array}$ \\
\hline$\omega \omega$ & $\begin{array}{l}\pi \\
K \\
\eta\end{array}$ & $\begin{array}{c}\frac{3}{2} g_{1} \\
\frac{1}{2}\left(g_{1}+g_{2}\right) \\
\frac{1}{6} g_{1}\end{array}$ & $\begin{array}{c}g_{3} \\
\frac{1}{3}\left(2 g_{3}-g_{5}\right) \\
\frac{1}{9} g_{3}\end{array}$ & $\begin{array}{c}\rho \\
K^{*} \\
\omega \\
\phi\end{array}$ & $\begin{array}{c}-2 g_{6}-\frac{7}{8} g_{8} \\
-\frac{3}{4} g_{6}+\frac{5}{12}\left(g_{7}-g_{8}\right)+\frac{1}{12} g_{9} \\
-\frac{2}{3} g_{6}-\frac{7}{24} g_{8} \\
0\end{array}$ \\
\hline$K^{*} K^{*}$ & $\begin{array}{l}\pi \\
K \\
\eta\end{array}$ & $\begin{array}{l}\frac{3}{8}\left(g_{1}+g_{2}\right) \\
\frac{3}{4}\left(g_{1}+g_{2}\right) \\
\frac{1}{24} g_{1}+\frac{3}{8} g_{2}\end{array}$ & $\begin{array}{l}\frac{1}{4}\left(2 g_{3}-g_{5}\right) \\
g_{3}-\frac{1}{2} g_{5} \\
\frac{5}{18} g_{3}-\frac{1}{4} g_{5}\end{array}$ & $\begin{array}{c}\rho \\
K^{*} \\
\omega \\
\phi\end{array}$ & $\begin{array}{c}-\frac{9}{16} g_{6}+\frac{5}{16}\left(g_{7}-g_{8}\right)+\frac{1}{16} g_{9} \\
-\frac{9}{8} g_{6}+\frac{5}{8}\left(g_{7}-g_{8}\right)+\frac{1}{8} g_{9} \\
-\frac{3}{16} g_{6}+\frac{5}{48}\left(g_{7}-g_{8}\right)+\frac{1}{48} g_{9} \\
-\frac{3}{8} g_{6}+\frac{5}{24}\left(g_{7}-g_{8}\right)+\frac{1}{24} g_{9}\end{array}$ \\
\hline$\phi \phi$ & $\begin{array}{l}\pi \\
K \\
\eta\end{array}$ & $\begin{array}{c}0 \\
g_{1}+g_{2} \\
\frac{2}{3} g_{1}\end{array}$ & $\begin{array}{c}0 \\
\frac{2}{3}\left(2 g_{3}-g_{5}\right) \\
\frac{4}{9} g_{3}\end{array}$ & $\begin{array}{c}\rho \\
K^{*} \\
\omega \\
\phi\end{array}$ & $\begin{array}{c}0 \\
-\frac{3}{2} g_{6}+\frac{5}{6}\left(g_{7}-g_{8}\right)+\frac{1}{6} g_{9} \\
0 \\
-\frac{4}{3} g_{6}-\frac{7}{12} g_{8}\end{array}$ \\
\hline$\omega \phi$ & $\begin{array}{l}\pi \\
K \\
\eta\end{array}$ & $\begin{array}{c}0 \\
\frac{1}{\sqrt{2}}\left(g_{1}-g_{2}\right) \\
0\end{array}$ & $\begin{array}{c}0 \\
\frac{\sqrt{2}}{3} g_{5} \\
0\end{array}$ & $\begin{array}{c}\rho \\
K^{*} \\
\omega \\
\phi\end{array}$ & $\begin{array}{c}-\frac{7}{6 \sqrt{2}} g_{6}-\frac{5}{6 \sqrt{2}}\left(g_{7}+g_{8}\right)-\frac{1}{6 \sqrt{2}} g_{9} \\
0 \\
0\end{array}$ \\
\hline
\end{tabular}

species of tadpole integrals may occur. We use the notations of [55] with $\bar{I}_{Q}^{(2)}$ and $\bar{I}_{Q}$, for which follows the infinite volume limit (17). For the finite volume case the standard result as, for instance, shown in [55] should be used. Note that analogous terms in the vector meson tadpoles are not resolved in this work. Here for typical QCD lattices the finite volume effects are negligible, being suppressed by factors $e^{-L M_{V}}$ with $L^{3}$ the volume of the considered box. The coefficient matrices $G_{V Q}^{(S)}$, $G_{V Q}^{(T)}$, and $G_{V R}^{(T)}$ are detailed in Table I.
There remain the bubble loop diagrams built in terms of the three-point vertices introduced in (5). The computation requires a further set of coupling constants $G_{Q P}^{(V)}, G_{R T}^{(V)}$, and $G_{Q R}^{(V)}$ that specify the strength of a three-point vertex in a given isospin projection. As in the previous section we use $P, Q \in[8]$ for the isospin multiplets of the Goldstone bosons and $V, R, T \in[9]$ for the ones of the vector mesons. The corresponding coefficients are collected in Table II. Our result,

TABLE II. The coupling constants for vector mesons $G_{Q R}^{(V)}$ with $V, R \in[9]$ and $P, Q \in[8]$ defined with respect to isospin states. Coupling constants that vanish due to $G$-parity considerations are not shown. It holds that $G_{P Q}^{(V)}=G_{Q P}^{(V)}$ and $G_{R T}^{(V)}=G_{T R}^{(V)}$.

\begin{tabular}{lll}
\hline \hline$G_{\pi \pi}^{(\rho)}=\frac{1}{\sqrt{3}} h_{1}$ & $G_{K K}^{(\rho)}=\frac{1}{\sqrt{6}} h_{1}$ & $G_{K K}^{(\omega)}=\frac{1}{\sqrt{6}} h_{1}$ \\
$G_{\pi K}^{\left(K^{*}\right)}=\frac{1}{\sqrt{8}} h_{1}$ & $G_{K \eta}^{\left(K^{*}\right)}=\frac{1}{\sqrt{8}} h_{1}$ & $G_{K K}^{(\phi)}=-\frac{1}{\sqrt{3}} h_{1}$ \\
$G_{\pi \omega}^{(\rho)}=2 \sqrt{\frac{2}{3}} h_{2}$ & $G_{\pi \rho}^{(\omega)}=2 \sqrt{2} h_{2}$ & $G_{\pi K^{*}}^{\left(K^{*}\right)}=\sqrt{2} h_{2}=G_{K \rho}^{\left(K^{*}\right)}$ \\
$G_{\eta \rho}^{(\rho)}=\frac{2}{3} \sqrt{2} h_{2}$ & $G_{\eta \omega}^{(\omega)}=\frac{2}{3} \sqrt{2} h_{2}$ & $G_{K \omega}^{\left(K^{*}\right)}=\sqrt{\frac{2}{3}} h_{2}$ \\
$G_{K K^{*}}^{(\rho)}=2 \sqrt{\frac{2}{3}} h_{2}$ & $G_{K K^{*}}^{(\omega)}=2 \sqrt{\frac{2}{3}} h_{2}$ & $G_{\eta K^{*}}^{\left(K^{*}\right)}=-\frac{\sqrt{2}}{3} h_{2}$ \\
$G_{K K^{*}}^{(\phi)}=\frac{4}{\sqrt{3}} h_{2}$ & $G_{\eta \phi}^{(\phi)}=-\frac{4}{3} \sqrt{2} h_{2}$ & $G_{K \phi}^{\left(K^{*}\right)}=\frac{2}{\sqrt{3}} h_{2}$ \\
$G_{\rho \rho}^{(\rho)}=6 \sqrt{2} h_{3}$ & $G_{K^{*} K^{*}}^{(\rho)}=6 h_{3}$ & $G_{K^{*} K^{*}}^{(\phi)}=-6 \sqrt{2} h_{3}$ \\
$G_{\rho K^{*}}^{\left(K^{*}\right)}=3 \sqrt{3} h_{3}$ & $G_{\omega K^{*}}^{\left(K^{*}\right)}=3 h_{3}$ & $G_{\phi K^{*}}^{\left(K^{*}\right)}=-3 \sqrt{2} h_{3}=-G_{K^{*} K^{*}}^{(\omega)} / \sqrt{2}$ \\
\hline \hline
\end{tabular}




$$
\begin{aligned}
\Pi_{V \in[9]}^{\text {bubble }}= & \sum_{Q, P \in[8]}\left(\frac{G_{Q P}^{(V)}}{2 f}\right)^{2}\left\{-\frac{1}{4}\left(m_{P}^{2}-m_{Q}^{2}\right)^{2} \Delta I_{Q P}-\frac{1}{4} M_{V}^{2}\left(\bar{I}_{Q}+\bar{I}_{P}\right)-\frac{1}{4} M_{V}^{2}\left(M_{V}^{2}-2\left(m_{P}^{2}+m_{Q}^{2}\right)\right) I_{Q P}\right\} \\
& +\sum_{Q \in[8], R \in[9]}\left(\frac{G_{Q R}^{(V)}}{2 f}\right)^{2}\left\{-\frac{1}{4} \alpha_{Q R}^{V}\left(M_{R}^{2}-m_{Q}^{2}\right)^{2} \Delta I_{Q R}-\frac{1}{4} \alpha_{Q R}^{V} M_{V}^{2} \bar{I}_{R}+\beta_{Q R}^{V} m_{Q}^{2} \bar{I}_{Q}+\delta_{Q R}^{V} \bar{I}_{Q}^{(2)}\right. \\
& \left.-\frac{1}{4} \alpha_{Q R}^{V} M_{V}^{2}\left(M_{V}^{2}-2\left(m_{Q}^{2}+M_{R}^{2}\right)\right) I_{Q R}\right\} \\
& +\sum_{R, T \in[9]}\left(\frac{G_{R T}^{(V)}}{2 f}\right)^{2}\left\{-\frac{1}{4} \alpha_{R T}^{V}\left(M_{R}^{2}-M_{T}^{2}\right)^{2} \Delta I_{R T}+\beta_{R T}^{V} M_{V}^{2} \bar{I}_{R}+\delta_{R T}^{V} M_{V}^{2} \bar{I}_{T}-\frac{1}{4} \alpha_{R T}^{V} M_{V}^{2}\left(M_{V}^{2}-2\left(M_{R}^{2}+M_{T}^{2}\right)\right) I_{R T}\right\} \\
\alpha_{Q R}^{V}= & \frac{\left(M_{R}^{2}+M_{V}^{2}\right)^{2}}{4 M_{R}^{2} M_{V}^{2}}, \quad \alpha_{R T}^{V}=M^{4} \frac{M_{R}^{2}+M_{V}^{2}+M_{T}^{2}}{3 M_{R}^{2} M_{V}^{2} M_{T}^{2}}, \quad \beta_{Q R}^{V}=-\frac{\left(M_{R}^{2}+M_{V}^{2}\right)^{2}+4 m_{Q}^{2}\left(M_{R}^{2}-M_{V}^{2}\right)}{16 M_{R}^{2} m_{Q}^{2}} \\
\beta_{R T}^{V}= & M^{4} \frac{8 M_{R}^{2}-M_{T}^{2}-M_{V}^{2}}{12 M_{R}^{2} M_{V}^{2} M_{T}^{2}}, \quad \delta_{Q R}^{V}=\frac{2 M_{R}^{2}+M_{V}^{2}}{8 M_{R}^{2}}, \quad \delta_{R T}^{V}=M^{4} \frac{8 M_{T}^{2}-M_{R}^{2}-M_{V}^{2}}{12 M_{R}^{2} M_{V}^{2} M_{T}^{2}},
\end{aligned}
$$

is expressed in terms of the tadpole integrals $\bar{I}_{Q}, \bar{I}_{P}$ and $\bar{I}_{R}, \bar{I}_{T}$ and the scalar bubble functions $I_{Q P}, \Delta I_{Q P}, I_{Q R}, \Delta I_{Q R}$ and $I_{R T}, \Delta I_{R T}$. We specify the generic case with $Q R$ for the infinite volume limit

$$
\begin{aligned}
& I_{Q R}=\bar{I}_{Q R}-\frac{\bar{I}_{R}}{M_{R}^{2}}, \quad \Delta I_{Q R}=I_{Q R}+\frac{\bar{I}_{Q}-\bar{I}_{R}}{m_{Q}^{2}-M_{R}^{2}} \\
& \bar{I}_{Q R}=\frac{1}{16 \pi^{2}}\left\{1-\frac{1}{2}\left(1+\frac{m_{Q}^{2}-M_{R}^{2}}{M_{V}^{2}}\right) \log \left(\frac{m_{Q}^{2}}{M_{R}^{2}}\right)+\frac{p_{Q R}}{M_{V}}\left(\log \left(1-\frac{M_{V}^{2}-2 p_{Q R} M_{V}}{m_{Q}^{2}+M_{R}^{2}}\right)-\log \left(1-\frac{M_{V}^{2}+2 p_{Q P} M_{V}}{m_{Q}^{2}+M_{R}^{2}}\right)\right)\right\} \\
& p_{Q R}^{2}=\frac{M_{V}^{2}}{4}-\frac{m_{Q}^{2}+M_{R}^{2}}{2}+\frac{\left(m_{Q}^{2}-M_{R}^{2}\right)^{2}}{4 M_{V}^{2}} .
\end{aligned}
$$

Corresponding expressions that hold for the scalar bubble in a finite box can be taken from [55]. The loop functions $I_{Q P}$ and $I_{R T}$ follow from (19) by appropriate replacements of the masses. Explicit expressions appropriate for the finite box case can be taken from [55].

The one-loop self-energy (18) will be analyzed in the following. In particular, its renormalization is scrutinized. We begin with the chiral limit of the vector meson masses. The reader may ask about the relevance of the chiral limit value, $M_{\chi}$, of the vector meson masses. After all, in this limit the phase space of the decay of a vector meson into pairs of Goldstone bosons is wide open and one may expect a significant decay width $\Gamma_{\chi}$. However, this is not the case. One readily obtains the expression

$$
\Gamma_{\chi}=\frac{h_{1}^{2} M_{\chi}^{3}}{2 \pi(16 f)^{2}} \simeq 0.24 \mathrm{GeV},
$$

where the numerical estimate is obtained with $M_{\chi}=$ $0.8 \mathrm{GeV}$ and $f=92 \mathrm{MeV}$. Such a value for the decay width seems compatible with our formal counting scheme $\Gamma_{\chi} \sim Q^{3}$ as compared to the scaling of the mass $M^{2} \sim Q^{2}$.

We turn to the vector meson mass in the chiral limit for which we obtain

$$
\begin{aligned}
M_{\chi}^{2}= & M^{2}+e_{1} M^{4}-\frac{3 M^{4}}{2 \pi^{2} f^{2}}\left(9 g_{6}-5 g_{7}+5 g_{8}-g_{9}\right) \log \frac{M^{2}}{\mu^{2}}-\frac{h_{1}^{2} M^{4}}{512 \pi^{2} f^{2}}\left(1-\log \frac{M^{2}}{\mu^{2}}\right)-\frac{7 h_{2}^{2} M^{4}}{144 \pi^{2} f^{2}} \log \frac{M^{2}}{\mu^{2}} \\
& +\frac{27 h_{3}^{2} M^{4}}{64 \pi^{2} f^{2}}\left(3-\sqrt{3} \pi+\log \frac{M^{2}}{\mu^{2}}\right)
\end{aligned}
$$

where we use $M_{\chi}$ for the renormalized mass of the vector mesons in the chiral limit. Here we replaced all vector meson masses $M_{V}, M_{R}, M_{T} \rightarrow M$ in (16) and (18) by the leading order expression. In addition, all masses of the
Goldstone bosons $m_{Q}, m_{P} \rightarrow 0$ are put to zero. The unknown parameter $e_{1}$ is needed to render the vector meson masses independent of the renormalization scale. All $\log \mu$ terms in (21) can be properly balanced by $e_{1}$. 
The result (21) is interesting since it gives a first hint of the importance of loop corrections to the vector meson masses. As expected, the loop corrections are suppressed in the large- $N_{c}$ limit of QCD. This is manifest in (21) with the scaling behavior $f^{2} \sim N_{c} ; M, g_{n}, h_{n} \sim N_{c}^{0}$; and $e_{1} \sim 1 / N_{c}$. Is this formal scaling property supported by corresponding numerical values at $N_{c}=3$ ? At the renormalization scale $\mu=M=0.80 \mathrm{GeV}$ and the particular parameter choices (9) we derive the estimate

$$
M_{\chi}^{2}-M^{2} \simeq e_{1} M^{4}-0.118 M^{2} \text { at } \mu=M,
$$

which does not depend on the so far unknown low-energy parameters $g_{n}$. The correction term (22) is comfortably small giving support to the assumed dimensional counting rules.

We consider a further physical quantity. The chiral limit of the $\omega-\phi$ mixing parameter is renormalized with

$$
\begin{aligned}
\left.\frac{1}{\sqrt{2}} \Pi_{\omega \phi}\right|_{m=0=m_{s}} & e_{2} M^{4}-\frac{M^{4}}{2 \pi^{2} f^{2}}\left(7 g_{6}+5 g_{7}+2 g_{8}+g_{9}\right) \log \frac{M^{2}}{\mu^{2}} \\
& +\frac{h_{1}^{2} M^{4}}{1536 \pi^{2} f^{2}}\left(1-\log \frac{M^{2}}{\mu^{2}}\right)-\frac{h_{2}^{2} M^{4}}{48 \pi^{2} f^{2}} \ln \left(\frac{M^{2}}{\mu^{2}}\right) \\
& -\frac{9 h_{3}^{2} M^{4}}{64 \pi^{2} f^{2}}\left(3-\sqrt{3} \pi+\log \frac{M^{2}}{\mu^{2}}\right),
\end{aligned}
$$

where we replaced again all vector meson masses $M_{V}, M_{R}$, $M_{T} \rightarrow M$ in (16) and (18) by the leading order expression [56]. In addition, all masses of the Goldstone bosons $m_{Q}$, $m_{P} \rightarrow 0$ are put to zero. The parameter $e_{2}$ makes the mixing angle scale invariant. The loop correction is suppressed by $1 / N_{c}$ as expected from the Okubo-Zweig-lizuka (OZI) rule. Even numerically we obtain with

$$
\left.\frac{1}{\sqrt{2}} \Pi_{\omega \phi}\right|_{m=0=m_{s}} \simeq e_{2} M^{4}+0.039 M^{2} \quad \text { at } \mu=M
$$

a small contribution to the mixing angle.

We continue with a study of the scale dependence of the remaining low-energy parameters. First we identify all scale-dependent terms in (16) and (18) that are proportional to $M^{2} m_{P, Q}^{2}$. Again $M_{V}, M_{R}, M_{T} \rightarrow M$ is used. Such terms define the running of the low-energy parameters $b_{i}$ as follows:

$\mu^{2} \frac{\mathrm{d}}{\mathrm{d} \mu^{2}} b_{1}=-\frac{9 h_{1}^{2}+8 h_{2}^{2}}{1536 \pi^{2} f^{2}}, \quad \mu^{2} \frac{\mathrm{d}}{\mathrm{d} \mu^{2}} b_{2}=-\frac{9 h_{1}^{2}+104 h_{2}^{2}}{4608 \pi^{2} f^{2}}$,

$\mu^{2} \frac{\mathrm{d}}{\mathrm{d} \mu^{2}} b_{3}=\frac{h_{1}^{2}-8 h_{2}^{2}}{256 \pi^{2} f^{2}}$.

For the symmetry breaking parameters $c_{i}$ we observe an interesting phenomenon. The renormalization scale invariance can be achieved only if specific correlations on the symmetry conserving low-energy parameters $g_{i}$ are imposed. This is seen as follows. A priori all scale dependent terms can be balanced only if we activate the $\mathrm{N}^{2} \mathrm{LO}$ and $\mathrm{N}^{3} \mathrm{LO}$ counterterms in (11), (12). We find

$$
\begin{aligned}
\mu^{2} \frac{\mathrm{d}}{\mathrm{d} \mu^{2}} c_{1}= & \frac{4 g_{1}-4 g_{2}+g_{5}}{192 \pi^{2} f^{2}}-\frac{h_{2}^{2}}{768 \pi^{2} f^{2}}, \\
\mu^{2} \frac{\mathrm{d}}{\mathrm{d} \mu^{2}} c_{2}= & \frac{44 g_{1}-20 g_{2}+6 g_{3}+5 g_{5}}{768 \pi^{2} f^{2}}-\frac{11 h_{2}^{2}}{3072 \pi^{2} f^{2}}, \\
M^{2} \mu^{2} \frac{\mathrm{d}}{\mathrm{d} \mu^{2}} c_{3}= & -\frac{68 g_{1}+36 g_{2}+26 g_{3}-9 g_{5}}{2304 \pi^{2} f^{2}} \\
& +\frac{17 h_{2}^{2}}{9216 \pi^{2} f^{2}}, \\
M^{2} \mu^{2} \frac{\mathrm{d}}{\mathrm{d} \mu^{2}} c_{4}= & -\frac{68 g_{1}+36 g_{2}+26 g_{3}-9 g_{5}}{1152 \pi^{2} f^{2}} \\
& +\frac{17 h_{2}^{2}}{4608 \pi^{2} f^{2}}, \\
M^{2} \mu^{2} \frac{\mathrm{d}}{\mathrm{d} \mu^{2}}\left(c_{5}+c_{6}\right)= & -\frac{4 g_{1}-4 g_{2}+g_{5}}{64 \pi^{2} f^{2}}+\frac{h_{2}^{2}}{256 \pi^{2} f^{2}}, \\
M^{4} \mu^{2} \frac{\mathrm{d}}{\mathrm{d} \mu^{2}} c_{7}= & \frac{4 g_{1}+g_{3}}{864 \pi^{2} f^{2}}-\frac{h_{2}^{2}}{3456 \pi^{2} f^{2}}, \\
M^{4} \mu^{2} \frac{\mathrm{d}}{\mathrm{d} \mu^{2}} c_{8}= & 0, \quad M^{2} \mu^{2} \frac{\mathrm{d}}{\mathrm{d} \mu^{2}} c_{9}=0 .
\end{aligned}
$$

From (26) we conclude that the hadrogenesis Lagrangian is renormalizable only if the following two sum rules

$$
4 g_{1}+g_{3}=\frac{1}{4} h_{2}^{2}, \quad g_{5}=g_{3}+4 g_{2}
$$

hold at leading order in the power counting scheme. In fact, we observe that once we insist on those two relations the leading order parameters $c_{1}$ and $c_{2}$ remain scale invariant.

In Fig. 1 we further scrutinize the numerical implications of our approach. We computed the polarization tensors for the four vector mesons as a function of the mass parameter $M$ for the particular renormalization scale $\mu=M$. The ratios $\Pi_{V}^{\text {loop }} / M^{2}$ are plotted in order to provide a direct measure for the importance of the loop effects. The physical vector meson masses are reproduced upon a suitable choice of the low-energy parameters determining the size of the tree-level contributions at order $Q^{4}$. Therefore the size of the plotted ratios is a direct measure for the naturalness of such low-energy parameters. Our results rely significantly on the consistency relations (27). As a consequence, there does not remain any residual dependence on any of the unknown parameters $g_{n}$. This is a particular property of the scenario with $\mu=M$.

Within the range of expected values $M_{\rho}<M<M_{\phi}$ all shown ratios are systematically smaller than one; however, 


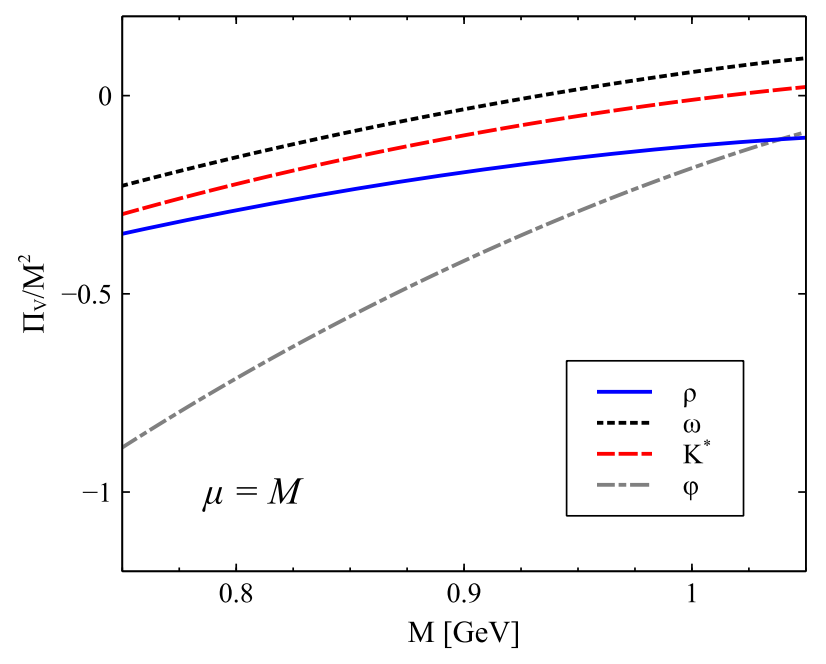

FIG. 1. The vector meson polarizations $\Pi_{V}^{\text {loop }} / M^{2}$ are presented as a function of $M$, at $\mu=M$. The plots rely on leading order bare masses for all mesons.

they are quite large for the $\phi$ meson at the lower bound of $M$. This together with the inverted pattern of the loop sizes for the different vector mesons would cause large low-energy parameters, possibly in conflict with the naturalness assumption. We note that the loop contributions are dominated largely by $h_{2}$ (see also [24,25,57]). The source of this effect is readily traced. It is a consequence of improperly approximated phase space factors $p_{Q R}^{2}$ using the replacement $M_{V, R, T} \rightarrow M$. This is a phenomenon known already from $\chi \mathrm{PT}$ studies of baryon masses $[55,58,59]$. An efficient remedy is the use of physical masses in the loop function [55,58-60]. The immediate concern is acknowledged: can any such scheme be scale invariant? In recent works $[59,60]$ a method was suggested that indeed leads to scale invariant results.

In the following we will adapt the formalism $[59,60]$ to our case at hand. In a first step we renormalize away all vector meson tadpole contributions with

$$
\begin{aligned}
\bar{I}_{R} & \rightarrow 0, \quad \bar{I}_{T} \rightarrow 0 \\
I_{Q R} & =\bar{I}_{Q R}-\frac{\bar{I}_{R}}{M_{R}^{2}} \rightarrow \bar{I}_{Q R}, \quad I_{R T}=\bar{I}_{R T}-\frac{1}{2}\left(\frac{\bar{I}_{R}}{M_{R}^{2}}+\frac{\bar{I}_{T}}{M_{T}^{2}}\right) \rightarrow \bar{I}_{R T} \\
I_{Q P} & =\bar{I}_{Q P}-\frac{\bar{I}_{V}}{M_{V}^{2}} \rightarrow \bar{I}_{Q P}
\end{aligned}
$$

where the residual objects $\bar{I}_{Q R}, \bar{I}_{R T}$, and $\bar{I}_{Q P}$ are scale invariant by construction. We emphasize that a subtraction scheme for the loop functions if performed at the level of the Passarino Veltman functions is symmetry conserving [55,58,61]. As long as there is an unambiguous prescription for how to represent all one-loop functions in terms of the Passarino Veltman functions we do not expect any violation of chiral Ward identities. In a second step we need to set up a power counting for physical masses. The crucial relations,

$$
\begin{aligned}
& m_{Q}^{2} \sim Q^{2}, \quad \frac{m_{Q}^{2}-m_{P}^{2}}{m_{Q}^{2}} \sim Q^{0}, \\
& M_{V}^{2} \sim Q^{2}, \quad \frac{M_{R}^{2}-M_{V}^{2}}{M_{R}^{2}} \sim Q^{2},
\end{aligned}
$$

are to be applied to all scale dependent terms. With (28) and (29) we obtain the result

$$
\begin{aligned}
\Pi_{V \in[9]}^{\text {bubble }}= & \sum_{Q, P \in[8]}\left(\frac{G_{Q P}^{(V)}}{2 f}\right)^{2}\left\{-\frac{1}{4}\left(m_{P}^{2}-m_{Q}^{2}\right)^{2} \Delta I_{Q P}-\frac{1}{4} M_{V}^{2}\left(\bar{I}_{Q}+\bar{I}_{P}\right)-\frac{1}{4} M_{V}^{2}\left(M_{V}^{2}-2\left(m_{P}^{2}+m_{Q}^{2}\right)\right) \bar{I}_{Q P}\right\} \\
& +\sum_{Q \in[8], R \in[9]}\left(\frac{G_{Q R}^{(V)}}{2 f}\right)^{2}\left\{-\frac{1}{4} \alpha_{Q R}^{V}\left(M_{R}^{2}-m_{Q}^{2}\right)^{2} \Delta I_{Q R}-\frac{1}{4} M_{V}^{2} \bar{I}_{Q}+\frac{3}{8} \bar{I}_{Q}^{(2)}-\frac{1}{4} \alpha_{Q R}^{V} M_{V}^{2}\left(M_{V}^{2}-2\left(m_{Q}^{2}+M_{R}^{2}\right)\right) \bar{I}_{Q R}\right\} \\
& +\sum_{R, T \in[9]}\left(\frac{G_{R T}^{(V)}}{2 f}\right)^{2}\left\{-\frac{1}{4} \alpha_{R T}^{V}\left(M_{R}^{2}-M_{T}^{2}\right)^{2} \Delta I_{R T}-\frac{1}{4} \alpha_{R T}^{V} M_{V}^{2}\left(M_{V}^{2}-2\left(M_{R}^{2}+M_{T}^{2}\right)\right) \bar{I}_{R T}\right\}+\mathcal{O}\left(Q^{6}\right) .
\end{aligned}
$$

We note that there are only three terms left that come with a scale dependence in (30). A simplification arises in the infinite volume limit with $m_{Q}^{2} \bar{I}_{Q} \rightarrow 4 \bar{I}_{Q}^{(2)}$. The term proportional to $\bar{I}_{Q}^{(2)}$ in (30) is canceled identically by corresponding terms in (16) if for the coupling constants $g_{1-5}$ our sum rules (27) are imposed. We consider the terms proportional to $\bar{I}_{Q}$ or $\bar{I}_{P}$ in (30). Their scale dependence can be balanced with

$$
\begin{aligned}
& \mu^{2} \frac{\mathrm{d}}{\mathrm{d} \mu^{2}} b_{1}^{\mathrm{r}}=-\frac{9 h_{1}^{2}+8 h_{2}^{2}}{4608 \pi^{2} f^{2}}, \quad \mu^{2} \frac{\mathrm{d}}{\mathrm{d} \mu^{2}} b_{2}^{\mathrm{r}}=-\frac{9 h_{1}^{2}+104 h_{2}^{2}}{13824 \pi^{2} f^{2}}, \\
& \mu^{2} \frac{\mathrm{d}}{\mathrm{d} \mu^{2}} b_{3}^{\mathrm{r}}=\frac{h_{1}^{2}-8 h_{2}^{2}}{768 \pi^{2} f^{2}},
\end{aligned}
$$

where we point at the factor changes in (31) as compared to (25). With $b_{i}^{\mathrm{r}}$ we denote the low-energy parameters that result in the scheme where the Passarino Veltman subtractions (28) are imposed. It is noted that, at first, such 
TABLE III. A rewrite of some terms in (13). With $M_{V} \rightarrow M$ and using the Gell-Mann-Oakes-Renner relations for the meson masses, the original expressions are recovered identically.

\begin{tabular}{lcc}
\hline \hline$V$ & $V=\rho$ & $V=\omega$ \\
\hline$M_{V}^{2} m_{\pi}^{2}$ & $23 \tilde{b}_{1}+3 \tilde{b}_{2}+9 \tilde{b}_{3}$ & $3\left(-3 \tilde{b}_{1}+\tilde{b}_{2}+35 \tilde{b}_{3}\right)$ \\
$M_{V}^{2} m_{K}^{2}$ & $4\left(\tilde{b}_{1}+\tilde{b}_{2}-\tilde{b}_{3}\right)$ & $4\left(\tilde{b}_{1}+\tilde{b}_{2}-\tilde{b}_{3}\right)$ \\
$M_{V}^{2} m_{\eta}^{2}$ & $-3 \tilde{b}_{1}+\tilde{b}_{2}+3 \tilde{b}_{3}$ & $-3 \tilde{b}_{1}+\tilde{b}_{2}+3 \tilde{b}_{3}$ \\
$V$ & $V=K^{*}$ & $V=\phi$ \\
$M_{V}^{2} m_{\pi}^{2}$ & $3\left(\tilde{b}_{1}+\tilde{b}_{2}-\tilde{b}_{3}\right)$ & $3\left(-3 \tilde{b}_{1}+\tilde{b}_{2}-13 \tilde{b}_{3}\right)$ \\
$M_{V}^{2} m_{K}^{2}$ & $4\left(3 \tilde{b}_{1}+\tilde{b}_{2}+5 \tilde{b}_{3}\right)$ & $4\left(5 \tilde{b}_{1}+\tilde{b}_{2}+11 \tilde{b}_{3}\right)$ \\
$M_{V}^{2} m_{\eta}^{2}$ & $9 \tilde{b}_{1}+\tilde{b}_{2}-9 \tilde{b}_{3}$ & $-3 \tilde{b}_{1}+\tilde{b}_{2}+51 \tilde{b}_{3}$ \\
\hline \hline
\end{tabular}

counterterms cancel the scale dependence only if the meson masses $m_{P}$ and $m_{Q}$ in (30) are replaced by their leading order representation as given by the Gell-Mann-OakesRenner (GOR) relations. In addition, the replacement $M_{V} \rightarrow M_{\chi}$ is needed. However, following the previous work $[59,60]$ we may recast the relevant quark mass terms in (13) into structures proportional to $m_{\pi}^{2}, m_{K}^{2}$, and $m_{\eta}^{2}$, where now the meson masses are not constrained by the Okubo relation $m_{\eta}^{2}=\left(4 m_{K}^{2}-m_{\pi}^{2}\right) / 3$ any longer. The particular form as detailed in Table III is dictated by the request of scale independence. Such a rewrite is unambiguous. It is readily constructed in terms of the convenient linear combinations

$\tilde{b}_{1}=\frac{6 b_{1}^{\mathrm{r}}-b_{3}^{\mathrm{r}}}{160}, \quad \tilde{b}_{2}=\frac{3 b_{2}^{\mathrm{r}}}{8}, \quad \tilde{b}_{3}=\frac{96 b_{1}^{\mathrm{r}}+144 b_{3}^{\mathrm{r}}}{7680}$,

with the scale dependence of $\tilde{b}_{1}$ and $\tilde{b}_{3}$ being determined by either $h_{2}$ or $h_{1}$. We assure the reader that with the rewrite of Table III our vector meson polarization tensors as implied by (30) are strictly scale invariant.

We wish to introduce yet a further additional subtraction for later convenience. With

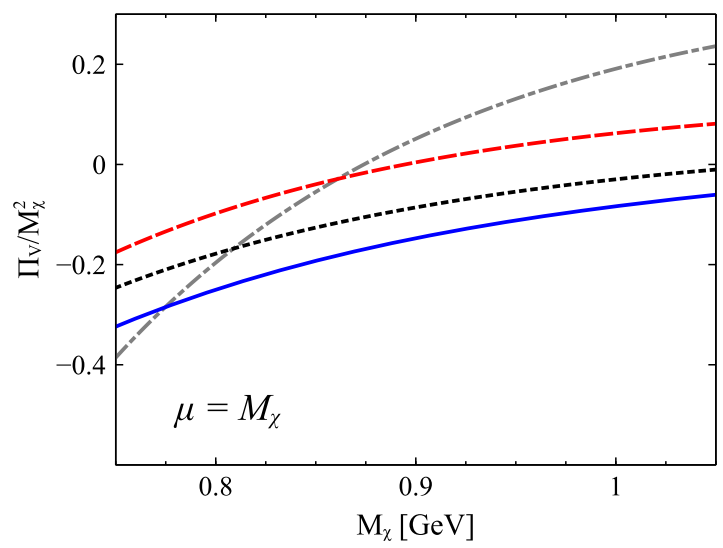

$\bar{I}_{Q P} \rightarrow \bar{I}_{Q P}-\frac{1}{(4 \pi)^{2}}, \quad \bar{I}_{R T} \rightarrow \bar{I}_{R T}-\frac{1-\pi / \sqrt{3}}{(4 \pi)^{2}}$,

we avoid any renormalization of the chiral limit mass value of the vector mesons. All low-energy parameters that result in the scheme where the Passarino Veltman subtractions (28) and (33) are applied receive an upper index ' $r$ ' to discriminate them from the bare parameters used initially. For instance, we have $M_{\chi}=M_{\mathrm{r}}$ and $e_{1}^{\mathrm{r}}=0$.

In the left-hand panel of Fig. 2 we show the ratios $\Pi_{V}^{\text {loop }} / M_{\chi}^{2}$ from (30). Here physical values for all meson masses together with the subtraction scheme (28) and (33) are scrutinized. The renormalization scale $\mu=M_{\chi}$ is identified with $M=M_{\chi}$. The ratios in Fig. 2 for the four vector mesons show an improved pattern as compared to the corresponding ratios of Fig. 1. The largest loop correction is obtained for the $\phi$ meson. All ratios are reasonably small implying natural sized counterterms.

Before providing a first numerical scenario for the set of low-energy constants, it should be mentioned that in the proposed scheme, where physical masses are used throughout all loop function, the $\omega-\phi$ mixing angle turns energy dependent necessarily. We deal with this situation by using two distinct mixing angles $\epsilon_{\omega}$ and $\epsilon_{\phi}$, which are introduced at the $\omega$ and $\phi$ masses, respectively. The mixing angles are then determined by the request that the transition polarization tensor

$$
\Pi_{\omega^{\prime} \phi^{\prime}}\left(s=m_{\omega}^{2}\right)=0=\Pi_{\omega^{\prime} \phi^{\prime}}\left(s=m_{\phi}^{2}\right),
$$

as computed in the prime basis, vanishes at the $\omega$ meson and the $\phi$ meson masses. These conditions determine the two mixing angles $\epsilon_{\omega}$ and $\epsilon_{\phi}$ in a self-consistent manner. The arising pattern for the mixing angles is anticipated with Fig. 3. In order to keep the renormalization scale invariance of our approach we need to recast the tree-level contribution into the following form:

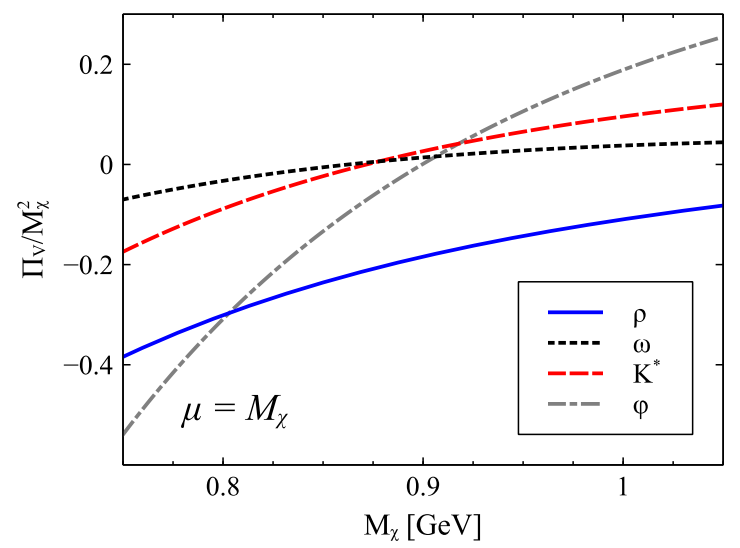

FIG. 2. The vector meson polarizations $\Pi_{V}^{\text {loop }} / M_{\chi}^{2}$ are presented as a function of $M_{\chi}$, at $\mu=M_{\chi}$, where physical values for all meson masses are assumed. While the left-hand plot show results at vanishing $\omega-\phi$ mixing angles, the right-hand plot illustrates the effect of nonvanishing $\omega-\phi$ mixing angles. Distinct values for the mixing angles at the $\omega$ and $\phi$ meson poles are assumed as explained in the text. 


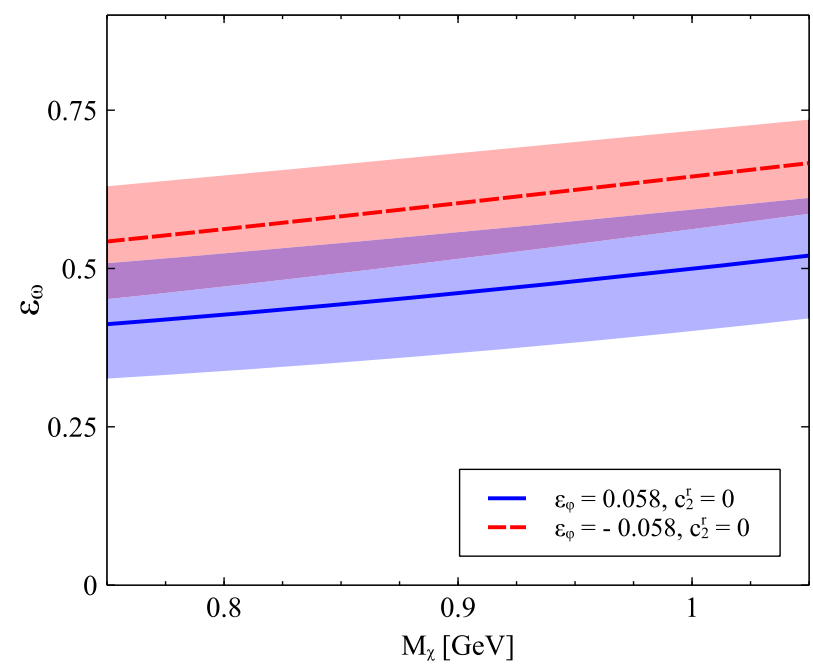

FIG. 3. The $\epsilon_{\omega}$ mixing angle derived from the two scenarios $\epsilon_{\phi}= \pm 0.058$. The low-energy parameters are adjusted to reproduce the physical meson masses. The bands indicate how $\epsilon_{\omega}$ changes when $c_{2}^{\mathrm{r}}$ varies from -1 to 1 .

$$
\Pi_{\omega \phi}^{\mathrm{tree}}(s)=\sqrt{2} e_{2}^{\mathrm{r}} s^{2}+\sqrt{2} b_{3}^{\mathrm{r}} s m_{K}^{2} .
$$

We emphasize that the tree-level and loop contributions to the mixing function $\Pi_{\omega^{\prime} \phi^{\prime}}(s)$ are evaluated with respect to the $\omega^{\prime}$ and $\phi^{\prime}$ fields. This is readily achieved in terms of the Clebsch coefficients in Tables I and II properly rotated into the $\omega^{\prime}, \phi^{\prime}$ basis. The case where mixing effects involve a vector meson propagating inside a loop contribution requires particular care. A scale invariant treatment arises only if terms proportional to any of the scalar bubble terms are rotated. This is readily justified since any residual tadpole term $\bar{I}_{Q}$ is not associated with an on-shell vector meson for which one can identify its corresponding mixing angle unambiguously.

Based on the scenario using physical meson masses we adjust the low-energy parameters to recover the physical vector meson masses. At a given value for $M$ we tune the parameters $b_{1}^{\mathrm{r}}, b_{2}^{\mathrm{r}}, b_{3}^{\mathrm{r}}$ together with $c_{1}^{\mathrm{r}}$. While the light quark mass $m$ is estimated from the empirical pion mass according to the GOR relation, the strange quark mass $m_{s}$ is determined by the empirical ratio $m_{s}=27.3 m$ [62]. The parameter $e_{2}^{\mathrm{r}}$ is set such that the value for the empirical mixing angle

$$
\epsilon_{\phi}= \pm 0.058
$$

as determined from the decay $\phi \rightarrow \pi_{0} \gamma$ in [8] arises. The parameter $c_{2}^{\mathrm{r}}$ is put to zero initially. Its determination requires further empirical input, as it may be provided from QCD lattice simulations of the vector meson masses at nonphysical quark masses. Note that the effect of $M_{\chi}$ and $c_{2}^{\mathrm{r}}$ on the vector meson masses can be discriminated only if data at various choices of the quark masses are considered.
We point out that our estimate for three-point coupling strength $h_{2}$ needs to be renormalized with

$$
h_{2} \simeq(2.33 \pm 0.03) / \cos \epsilon_{\omega},
$$

since its previous estimate rests on the decay process $\omega \rightarrow \rho \pi$ analyzed in the absence of mixing effects $[13,14]$.

In Fig. 3 we present our result for the mixing angle $\epsilon_{\omega}$ as it results from a fit to the physical masses as described above. We observe a significant energy dependence of the loop contribution to $\Pi_{\omega \phi}^{\text {loop}}$, in line with the conclusions from previous works $[25,28]$. However, we would argue that a proper treatment of the $\omega-\phi$ mixing phenomenon requires a two mixing angle scenario: while the mixing angle may be as small as $\epsilon=\epsilon_{\phi}= \pm 0.058$ at the $\phi$ meson mass, at the $\omega$ meson mass the mixing angle $\epsilon=\epsilon_{\omega}$ is an order of magnitude larger. We checked with the bandwidths in Fig. 3 that variations of the form $-1<c_{2}^{\mathrm{r}}<1$ do not change this spectacular pattern. It may not come as a surprise that such a large mixing phenomenon does mend the form of the loop contributions to the vector meson masses. Indeed, as shown in the right-hand plot of Fig. 2 the size of the polarization tensor for the $\omega$ meson is affected significantly as compared to the left-hand plot of the same figure that uses $\epsilon_{\omega}=\epsilon_{\phi}=0$.

In Fig. 4 we show the result for the low-energy parameters in a given range of $M_{\chi}$, using physical masses. Note that these were already used in Fig. 3. For all parameters we obtain naturally sized values.

\section{GOLDSTONE BOSONS AT THE ONE-LOOP LEVEL}

We first collect all tree-level contributions to the pseudoscalar meson masses as implied by $\mathcal{L}_{2}^{(2)}$ and $\mathcal{L}_{4}^{(2)}$. The well-known expressions first derived by Gasser and Leutwyler [51,63] are obtained:

$$
\begin{aligned}
m_{\pi}^{2}= & 2 B_{0} m+\frac{32 B_{0}^{2} m}{f^{2}}\left[\left(2 m+m_{s}\right)\left(2 L_{6}-L_{4}\right)\right. \\
& \left.+m\left(2 L_{8}-L_{5}\right)\right]+\cdots, \\
m_{K}^{2}= & B_{0}\left(m+m_{s}\right)+\frac{16 B_{0}^{2}\left(m+m_{s}\right)}{f^{2}}\left[\left(2 m+m_{s}\right)\left(2 L_{6}-L_{4}\right)\right. \\
& \left.+\frac{1}{2}\left(m+m_{s}\right)\left(2 L_{8}-L_{5}\right)\right]+\cdots, \\
m_{\eta}^{2}= & \frac{2}{3} B_{0}\left(m+2 m_{s}\right)+\frac{128 B_{0}^{2}}{9 f^{2}}\left(m-m_{s}\right)^{2}\left(3 L_{7}+L_{8}\right) \\
& +\frac{32 B_{0}^{2}\left(m+2 m_{s}\right)}{3 f^{2}}\left[\left(2 m+m_{s}\right)\left(2 L_{6}-L_{4}\right)\right. \\
& \left.+\frac{1}{3}\left(m+2 m_{s}\right)\left(2 L_{8}-L_{5}\right)\right]+\cdots .
\end{aligned}
$$



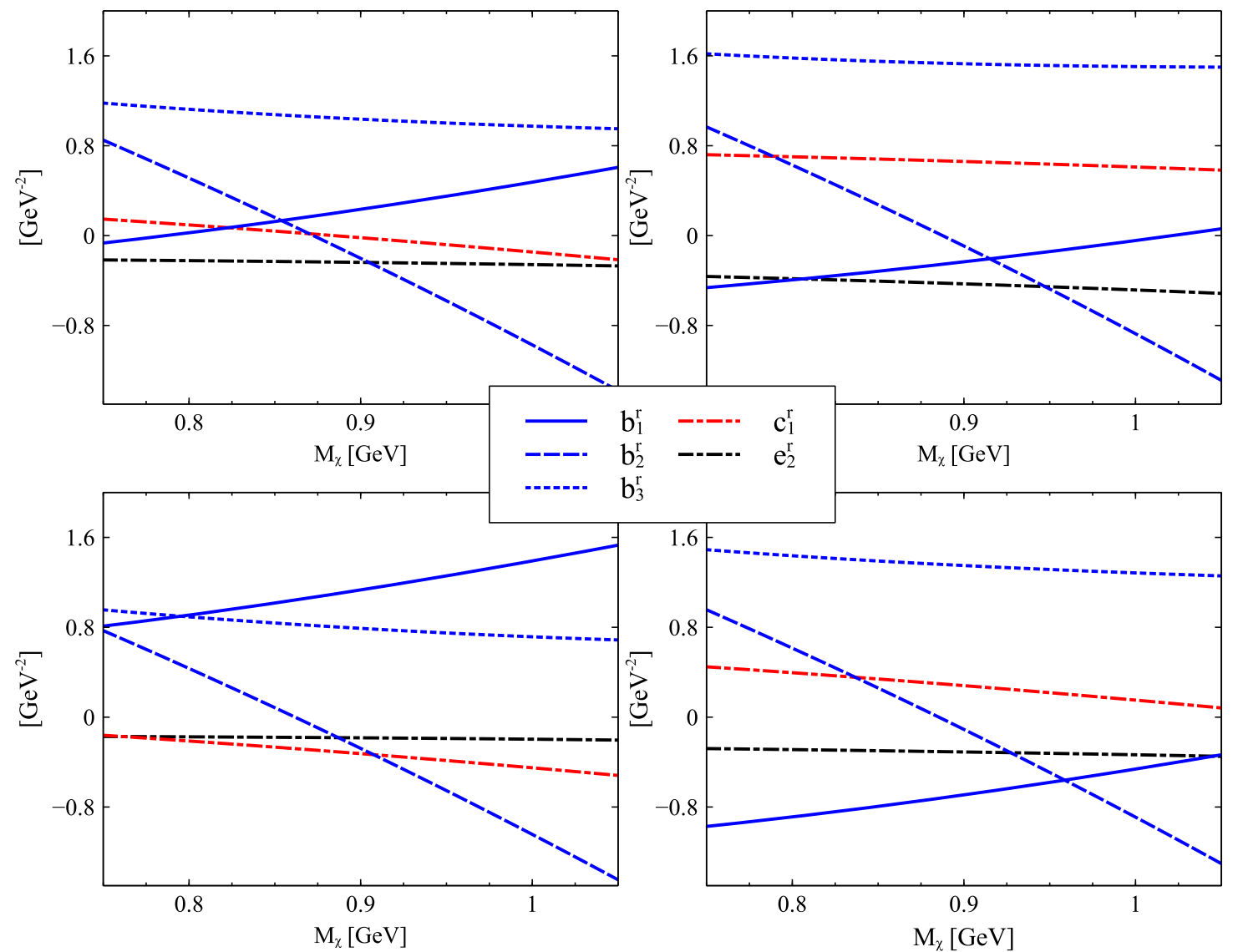

FIG. 4. The result for the low-energy parameters for a natural range of $M_{\chi}$ with $\mu=M_{\chi}=M_{\mathrm{r}}$. Physical masses are used. While the upper plots are with $c_{2}^{\mathrm{r}}=0$ where $\epsilon_{\phi}=0.058$ (left) and $\epsilon_{\phi}=-0.058$ (right), the lower ones follow with $\epsilon_{\phi}=0.058$ where $c_{2}^{\mathrm{r}}=-1$ (left) and $c_{2}^{\mathrm{r}}=1$ (right).

At the one-loop level there are, in addition, tadpole-type contributions. The terms involving the tadpole of the pseudoscalar mesons were considered already in [51,63].
In contrast, corresponding structures involving the tadpole with vector mesons are less well studied. Altogether we find

TABLE IV. The coupling constants for vector mesons $G_{P Q}^{(\chi)}, G_{P Q}^{(T)}$, and $G_{P V}^{(T)}$ with $V \in[9]$ and $P, Q \in[8]$ defined with respect to isospin states.

\begin{tabular}{|c|c|c|c|c|c|}
\hline$P$ & $Q$ & $G_{P Q}^{(\chi)}$ & $G_{P Q}^{(T)}$ & $V$ & $G_{P V}^{(T)}$ \\
\hline$\pi$ & $\begin{array}{l}\pi \\
K \\
\eta\end{array}$ & $\begin{aligned}(20 / 3) B_{0} m & \rightarrow(28 / 9) m_{\pi}^{2}+(8 / 9) m_{K}^{2}-(2 / 3) m_{\eta}^{2} \\
(4 / 3) B_{0}\left(3 m+m_{s}\right) & \rightarrow(4 / 3)\left(m_{\pi}^{2}+m_{K}^{2}\right) \\
(4 / 3) B_{0} m & \rightarrow(2 / 3) m_{\pi}^{2}\end{aligned}$ & $\begin{array}{c}-\frac{8}{3} \\
-\frac{4}{3} \\
0\end{array}$ & $\begin{array}{c}\rho \\
K^{*} \\
\omega \\
\phi\end{array}$ & $\begin{array}{c}2 g_{1}+4 g_{2}+\frac{3}{2} g_{3}-g_{5} \\
2 g_{1}+2 g_{2}+g_{3}-\frac{1}{2} g_{5} \\
2 g_{1}+\frac{1}{2} g_{3} \\
0\end{array}$ \\
\hline$K$ & $\begin{array}{l}\pi \\
K \\
\eta\end{array}$ & $\begin{array}{c}B_{0}\left(3 m+m_{s}\right) \rightarrow m_{\pi}^{2}+m_{K}^{2} \\
4 B_{0}\left(m+m_{s}\right) \rightarrow(2 / 3)\left(3 m_{\eta}^{2}+2 m_{K}^{2}+m_{\pi}^{2}\right) \\
(1 / 3) B_{0}\left(m+3 m_{s}\right) \rightarrow m_{\eta}^{2}-(1 / 3) m_{K}^{2}\end{array}$ & $\begin{array}{l}-1 \\
-2 \\
-1\end{array}$ & $\begin{array}{c}\rho \\
K^{*} \\
\omega \\
\phi\end{array}$ & $\begin{array}{c}\frac{3}{2} g_{1}+\frac{3}{2} g_{2}+\frac{3}{4} g_{3}-\frac{3}{8} g_{5} \\
3 g_{1}+3 g_{2}+\frac{3}{2} g_{3}-\frac{3}{4} g_{5} \\
\frac{1}{2} g_{1}+\frac{1}{2} g_{2}+\frac{1}{4} g_{3}-\frac{1}{8} g_{5} \\
g_{1}+g_{2}+\frac{1}{2} g_{3}-\frac{1}{4} g_{5}\end{array}$ \\
\hline$\eta$ & $\begin{array}{l}\pi \\
K \\
\eta\end{array}$ & $\begin{array}{c}4 B_{0} m \rightarrow 2 m_{\pi}^{2} \\
(4 / 3) B_{0}\left(m+3 m_{s}\right) \rightarrow 4 m_{\eta}^{2}-\left(4 m_{K}^{2}\right) / 3 \\
(4 / 9) B_{0}\left(m+8 m_{s}\right) \rightarrow(2 / 3)\left(7 m_{\eta}^{2}-4 m_{K}^{2}\right)\end{array}$ & $\begin{array}{c}0 \\
-4 \\
0\end{array}$ & $\begin{array}{c}\rho \\
K^{*} \\
\omega \\
\phi\end{array}$ & $\begin{array}{c}2 g_{1}+\frac{1}{2} g_{3} \\
\frac{2}{3} g_{1}+6 g_{2}+\frac{5}{3} g_{3}-\frac{3}{2} g_{5} \\
\frac{2}{3} g_{1}+\frac{1}{6} g_{3} \\
\frac{8}{3} g_{1}+\frac{2}{3} g_{3}\end{array}$ \\
\hline
\end{tabular}




$$
\begin{aligned}
\Pi_{P \in[8]}^{\mathrm{tadpole}}= & \frac{1}{4 f^{2}} \sum_{Q \in[8]}\left\{-\left(m_{P}^{2}+m_{Q}^{2}\right) G_{P Q}^{(T)}-G_{P Q}^{(\chi)}\right\} \bar{I}_{Q} \\
& +\frac{3}{4 f^{2}} \sum_{V \in[9]}\left\{-2 m_{P}^{2} G_{P V}^{(T)}\right\} \bar{I}_{V},
\end{aligned}
$$

with the tadpole function $\bar{I}_{Q}$ already recalled in (17). The vector meson tadpole $\bar{I}_{V}$ follows from $\bar{I}_{Q}$ with the replacement $m_{Q} \rightarrow M_{V}$. The coefficients $G_{P Q}^{(T)}, G_{P Q}^{(\chi)}$, and $G_{P V}^{(T)}$ are detailed in Table IV. The index $P, Q \in[8]$ runs over the octet of Goldstone bosons, properly grouped into isospin multiplets. The index $V \in[9]$ runs over the nonet of vector mesons. While the parameters $B_{0}$ and $m, m_{s}$ determine $G_{P Q}^{(T)}, G_{P Q}^{(\chi)}$, the additional vector meson parameters $g_{1-5}$ are probed in $G_{P V}^{(T)}$.

At the one-loop level there remain additional contributions involving vertices from $\mathcal{L}_{2}^{(3)}$ in (5), which involve a bubble loop integral. We derive their form with

$$
\begin{aligned}
\Pi_{P \in[8]}^{\text {bubble }}= & \sum_{Q \in[8], V \in[9]}\left(\frac{G_{Q V}^{(P)}}{2 f}\right)^{2}\left\{-\frac{1}{4}\left(M_{V}^{2}-m_{Q}^{2}\right)^{2} \Delta I_{Q V}-\frac{1}{4} m_{P}^{2}\left(\bar{I}_{Q}+\bar{I}_{V}\right)-\frac{1}{4} m_{P}^{2}\left(m_{P}^{2}-2\left(m_{Q}^{2}+M_{V}^{2}\right)\right) I_{Q V}\right\} \\
& +\sum_{V, R \in[9]}\left(\frac{G_{V R}^{(P)}}{2 f}\right)^{2}\left\{-\frac{1}{4} \alpha_{V R}^{P}\left(M_{R}^{2}-M_{V}^{2}\right)^{2} \Delta I_{V R}+\left(\beta_{V R}^{P} m_{P}^{2} \bar{I}_{V}+\beta_{R V}^{P} m_{P}^{2} \bar{I}_{R}\right)-\frac{1}{4} \alpha_{V R}^{P} m_{P}^{2}\left(m_{P}^{2}-2\left(M_{R}^{2}+M_{V}^{2}\right)\right) I_{V R}\right\}, \\
\alpha_{V R}^{P}= & \frac{\left(M_{V}^{2}+M_{R}^{2}\right)^{2}}{4 M_{V}^{2} M_{R}^{2}}, \quad \beta_{V R}^{P}=\frac{7 M_{V}^{4}-10 M_{V}^{2} M_{R}^{2}-2 M_{R}^{4}}{32 M_{V}^{2} M_{R}^{2}},
\end{aligned}
$$

in terms of the scalar bubble functions $I_{Q V}, \Delta I_{Q V}, I_{V R}$, $\Delta I_{V R}$ and the previously introduced tadpole integrals $\bar{I}_{Q}$, $\bar{I}_{V}, \bar{I}_{R}$. The loop functions $I_{Q V}$ follow from $I_{Q R}$ in (19) with the replacements $M_{V} \rightarrow m_{P}$ together with $M_{R} \rightarrow M_{V}$. In contrast, the loop functions $I_{V R}$ follow from $I_{Q V}$ with the replacement $m_{Q} \rightarrow M_{R}$. For later convenience and in order to avoid any misinterpretation, we provide the explicit representation nevertheless,

$$
\begin{aligned}
I_{V R}= & \bar{I}_{V R}-\frac{\bar{I}_{R}}{2 M_{R}^{2}}-\frac{\bar{I}_{V}}{2 M_{V}^{2}}, \quad \Delta I_{V R}=I_{V R}+\frac{\bar{I}_{V}-\bar{I}_{R}}{M_{V}^{2}-M_{R}^{2}}, \\
\bar{I}_{V R}= & \frac{1}{16 \pi^{2}}\left\{1-\frac{M_{V}^{2}-M_{R}^{2}}{2 m_{P}^{2}} \log \left(\frac{M_{V}^{2}}{M_{R}^{2}}\right)\right. \\
& +\frac{p_{V R}}{m_{P}}\left(\log \left(1-\frac{m_{P}^{2}-2 p_{V R} m_{P}}{M_{V}^{2}+M_{R}^{2}}\right)\right. \\
& \left.\left.-\log \left(1-\frac{m_{P}^{2}+2 p_{V P} m_{P}}{M_{V}^{2}+M_{R}^{2}}\right)\right)\right\}, \\
p_{V R}^{2}= & \frac{m_{P}^{2}}{4}-\frac{M_{V}^{2}+M_{R}^{2}}{2}+\frac{\left(M_{V}^{2}-M_{R}^{2}\right)^{2}}{4 m_{P}^{2}},
\end{aligned}
$$

where we point at the symmetric definition of the object $\bar{I}_{V R}=\bar{I}_{R V}$. The coefficients $G_{Q R}^{(P)}$ and $G_{V R}^{(P)}$ with $P, \quad Q \in[8]$ and $V, \quad R \in[9]$ are proportional to the coupling constants $h_{1}$ and $h_{2}$. They are listed in Table V.

A few comments are in order here. All contributions in (39) and (40) comply with their expected power counting order $Q^{4}$ in the scenario where $m_{P, Q} \sim M_{V, R} \sim Q$. The scale dependence from the loop contributions is balanced by a corresponding dependence of the low-energy constants. In order to establish a strict renormalization we have to decompose the lowenergy constant $B_{0}$ into its power counting moments with

$$
B_{0}=\sum_{n=0} M^{2 n} B_{0}^{(2 n)}
$$

This implies the condition

TABLE V. The coupling constants $G_{Q R}^{(P)}$ and $G_{V R}^{(P)}=G_{R V}^{(P)}$ with $P, Q \in[8]$ and $V, R \in[9]$.

\begin{tabular}{llll}
\hline \hline$G_{\pi \rho}^{(\pi)}=\sqrt{2} h_{1}$ & $G_{K K^{*}}^{(\pi)}=h_{1}$ & $G_{\pi K^{*}}^{(K)}=-\frac{\sqrt{3}}{2} h_{1}$ & $G_{K \rho}^{(K)}=\frac{\sqrt{3}}{2} h_{1}$ \\
$G_{K \omega}^{(K)}=\frac{1}{2} h_{1}$ & $G_{\eta K^{*}}^{(K)}=-\frac{\sqrt{3}}{2} h_{1}$ & $G_{K \phi}^{(K)}=-\frac{\sqrt{2}}{2} h_{1}$ & $G_{K K^{*}}^{(\eta)}=\sqrt{3} h_{1}$ \\
$G_{\rho \omega}^{(\pi)}=2 h_{2}$ & $G_{\rho K^{*}}^{(K)}=\sqrt{3} h_{2}$ & $G_{\rho \rho}^{(\eta)}=2 h_{2}$ & $G_{K^{*} K^{*}}^{(\eta)}=-\frac{2}{\sqrt{3}} h_{2}$ \\
$G_{K^{*} K^{*}}^{(\pi)}=2 h_{2}$ & $G_{\omega K^{*}}^{(K)}=G_{\phi K^{*}}^{(K)} / \sqrt{2}=h_{2}$ & $G_{\omega \omega}^{(\eta)}=\frac{2}{\sqrt{3}} h_{2}$ & $G_{\phi \phi}^{(\eta)}=-\frac{4}{\sqrt{3}} h_{2}$ \\
\hline \hline
\end{tabular}




$$
\begin{aligned}
\mu^{2} \frac{\mathrm{d}}{\mathrm{d} \mu^{2}} B_{0}^{(2)}= & -\frac{9\left(4 g_{1}+4 g_{2}+2 g_{3}-g_{5}\right)}{64 \pi^{2} f^{2}} B_{0}^{(0)} \\
& -\frac{9 h_{1}^{2}}{256 \pi^{2} f^{2}} B_{0}^{(0)}-\frac{63 h_{2}^{2}}{256 \pi^{2} f^{2}} B_{0}^{(0)}, \\
\mu^{2} \frac{\mathrm{d}}{\mathrm{d} \mu^{2}}\left(2 L_{6}-L_{4}\right)= & -\frac{1}{1152 \pi^{2}}-\frac{3 h_{1}^{2}}{4096 \pi^{2}}, \\
\mu^{2} \frac{\mathrm{d}}{\mathrm{d} \mu^{2}}\left(3 L_{7}+L_{8}\right)= & -\frac{5}{1536 \pi^{2}}, \\
\mu^{2} \frac{\mathrm{d}}{\mathrm{d} \mu^{2}}\left(2 L_{8}-L_{5}\right)= & +\frac{1}{192 \pi^{2}}-\frac{3 h_{1}^{2}}{4096 \pi^{2}}+\frac{3 h_{2}^{2}}{512 \pi^{2}} .
\end{aligned}
$$

In order to scrutinize the importance of dynamical vector meson degrees of freedom, it is useful to match our results to the conventional $\chi \mathrm{PT}$ expression derived from the flavor SU(3) Lagrangian at the one-loop level. This is readily achieved by a further chiral expansion of (39) and (40) where now the counting rule $m_{Q} / m_{V} \sim Q$ has to be applied. If truncated to order $Q^{4}$ the only effect of the vector mesons is a renormalization of Gasser and Leutwyler's low-energy constants. With this we find

$$
\begin{aligned}
B_{0}^{\text {ren }} & =B_{0}-\frac{9 B_{0}\left(4 g_{1}+4 g_{2}+2 g_{3}-g_{5}\right)}{64 \pi^{2} f^{2}} M^{2} \log \frac{M^{2}}{\mu^{2}}-\frac{3 h_{1}^{2} M^{2} B_{0}}{512 \pi^{2} f^{2}}\left(1+6 \log \frac{M^{2}}{\mu^{2}}\right)-\frac{3 h_{2}^{2} M^{2} B_{0}}{256 \pi^{2} f^{2}}\left(16+21 \log \frac{M^{2}}{\mu^{2}}\right), \\
2 L_{6}^{\text {ren }}-L_{4}^{\text {ren }} & =2 L_{6}-L_{4}-\frac{h_{1}^{2}}{8192 \pi^{2}}\left(1+6 \log \frac{M^{2}}{\mu^{2}}\right), \\
2 L_{8}^{\text {ren }}-L_{5}^{\text {ren }} & =2 L_{8}-L_{5}-\frac{h_{1}^{2}}{8192 \pi^{2}}\left(-7+6 \log \frac{M^{2}}{\mu^{2}}\right)-\frac{h_{2}^{2}}{4096 \pi^{2}}\left(-40-24 \log \frac{M^{2}}{\mu^{2}}\right), \\
3 L_{7}^{\text {ren }}+L_{8}^{\text {ren }} & =3 L_{7}+L_{8} .
\end{aligned}
$$

The contributions proportional to $h_{1}^{2}$ have been considered in the literature before $[4,6,7,11,44,45]$. Our results are consistent with the recent study $[44,45]$. The effect of the coupling constant $h_{2}$ is typically not considered in resonance saturation approaches to Gasser and Leutwyler's low-energy constants $[4,6,7,47]$. In particular, its contribution to $B_{0}$ and $2 L_{8}-L_{5}$ is sizable, a factor of 10-30 larger than the corresponding terms proportional to $h_{1}^{2}$. Again it is convenient to explore the size of the loop effects at the particular renormalization scale $\mu=M$. In this case we obtain

$$
B_{0}^{\text {ren }} / B_{0}=1-\underbrace{3\left(h_{1}^{2} / 32+h_{2}^{2}\right)}_{\simeq 16.9}\left(\frac{M}{4 \pi f}\right)^{2},
$$

a huge correction term primarily caused by the $h_{2}$ term. In contrast to our findings in the vector meson sector, we observe a significant size of the loop correction that poses a challenge to the dimensional counting rules.

Note, however, that the result (45) is not unexpected since the typical ratio

$$
\frac{M}{\Lambda_{\chi}}=\frac{M}{4 \pi f} \sim 1
$$

is probed in (44), which is of order one numerically in any case. While for sufficiently large values of $N_{c}$ we have $\Lambda_{\chi} \geq \Lambda_{\mathrm{HG}}$ by assumption, this is not the case for the physical choice with $N_{c}=3$ and $\Lambda_{\chi} \simeq 1 \mathrm{GeV}$. In turn all terms proportional to $\left(M / \Lambda_{\chi}\right)^{n}$ need to be summed in our approach. This is the target of the following development. We wish to identify renormalized low-energy parameters $l^{\text {ren }}$ that have a decomposition of the following form:

$$
l^{\text {ren }}=\sum_{n=0}^{\infty} l_{n}\left(\frac{M}{4 \pi f}\right)^{2 n} \quad \text { with } \quad l^{\text {ren }} \sim \Lambda_{\mathrm{HG}}^{\operatorname{dim}[\operatorname{lren}]},
$$

and therefore justify the application of the dimensional counting rules. While for sufficiently large $N_{c}$ the renormalized coupling constants can be conveniently matched to the parameters of the hadrogenesis Lagrangian in perturbation theory, at $N_{c}=3$ a suitable summation scheme is required. Such a scheme is readily devised by exploiting the simple observation: the particular combination

$$
\left(\frac{M}{4 \pi f}\right)^{2} \frac{B_{0} m_{\text {quark }}}{M^{2}} \sim Q^{2}
$$

is consistent with the dimensional counting rule the hadrogenesis Lagrangian is based on. Note that the second factor in (48) arises naturally if a loop contribution involving vector mesons is expanded in powers of the quark masses. We conclude that if we absorb any terms proportional to powers of the ratio $M / \Lambda_{\chi}$ into the low-energy parameters of the chiral Lagrangian, then necessarily the particular combination (48) arises. For instance, in the chiral domain it followed for the accordingly renormalized loop contribution 


$$
\left.\Pi_{P}^{\text {bubble }}\right|_{\text {renormalized }} \sim B_{0}^{2} m_{\text {quark }}^{2}
$$

for sufficiently small quark masses. All nonperturbative effects in $M / \Lambda_{\chi}$ are moved into the renormalized lowenergy parameters. The important observation is that there is no need to actually perform the infinite summation explicitly. Since such a summation should be performed in accordance with the symmetries of the hadrogenesis Lagrangian, the generic structure of the result must resemble the generic structure of a perturbative computation at $\Lambda_{\chi} \geq \Lambda_{\mathrm{HG}}$. Thus it suffices to express the bare coupling constants in terms of the renormalized coupling constants order by order in perturbation theory. Technically it is more economical to devise a suitable subtraction scheme for the loop functions involving vector mesons $[55,58,61]$. If performed at the level of the Passarino Veltman functions, such a renormalization scheme is symmetry conserving and not violating any chiral Ward identities.

We introduce the following subtraction rules:

$$
\begin{aligned}
\bar{I}_{V} & \rightarrow 0, \quad \bar{I}_{R} \rightarrow 0, \\
I_{Q V} & \rightarrow \bar{I}_{Q V}+\frac{1}{4} \frac{1}{(4 \pi)^{2}}, \quad I_{V R} \rightarrow \bar{I}_{V R}+\frac{1}{(4 \pi)^{2}},
\end{aligned}
$$

where all low-energy parameters within the renormalization scheme (50) receive an upper index ' $r$ ' as to discriminate them from the bare parameters used initially. Indeed, in (39) and (40) all contributions from the vector meson loops to the low-energy parameter $B_{0}^{\mathrm{r}}$ vanish identically. Note that the scale invariant bubble structures $\Delta I_{Q V}$ and $\Delta I_{V R}$ remain untouched. Given the subtraction scheme (50) it is feasible to use physical meson masses everywhere without picking up an uncontrolled dependence on the renormalization scale $\mu$. As for the vector meson polarization tensor it suffices to reinterpret the appropriate counterterm contributions

$$
\begin{aligned}
m_{\pi}^{2}= & 2 B_{0}^{\mathrm{r}} m-\frac{8 m_{\pi}^{2}}{f^{2}}\left[m_{\pi}^{2}\left(L_{5}^{\mathrm{r}}-2 L_{8}^{\mathrm{r}}\right)\right. \\
& \left.+\left(2 m_{K}^{2}+m_{\pi}^{2}\right)\left(L_{4}^{\mathrm{r}}-2 L_{6}^{\mathrm{r}}\right)\right]+\cdots, \\
m_{K}^{2}= & B_{0}^{\mathrm{r}}\left(m+m_{s}\right)-\frac{8 m_{K}^{2}}{f^{2}}\left[m_{K}^{2}\left(L_{5}^{\mathrm{r}}-2 L_{8}^{\mathrm{r}}\right)\right. \\
& \left.+\frac{3}{2}\left(m_{\eta}^{2}+m_{\pi}^{2}\right)\left(L_{4}^{\mathrm{r}}-2 L_{6}^{\mathrm{r}}\right)\right]+\cdots, \\
m_{\eta}^{2}= & \frac{2}{3} B_{0}^{\mathrm{r}}\left(m+2 m_{s}\right)-\frac{8 m_{\eta}^{2}}{f^{2}}\left[m_{\eta}^{2}\left(L_{5}^{\mathrm{r}}-2 L_{8}^{\mathrm{r}}\right)\right. \\
& \left.-3\left(m_{\eta}^{2}-2 m_{K}^{2}\right)\left(L_{4}^{\mathrm{r}}-2 L_{6}^{\mathrm{r}}\right)\right] \\
& +\frac{128}{40 f^{2}}\left[13 m_{\eta}^{4}-8 m_{\eta}^{2} m_{K}^{2}-8 m_{K}^{4}+3 m_{\pi}^{4}\right]\left(L_{8}^{\mathrm{r}}+3 L_{7}^{\mathrm{r}}\right) \\
& +\cdots,
\end{aligned}
$$

in terms of physical masses. Note that this result was derived already in [59]. We assure the reader that with (51) and the replacements provided in Table IV scale invariant results for the pion, kaon, and eta meson masses are obtained.

The subtraction scheme implies, in particular, that there remains no explicit dependence on any of the unknown low-energy parameters $g_{n}$. Moreover, a modification for the expression for the renormalized lowenergy constants with

$\mu^{2} \frac{\mathrm{d}}{\mathrm{d} \mu^{2}}\left(2 L_{6}^{\mathrm{r}}-L_{4}^{\mathrm{r}}\right)=-\frac{1}{1152 \pi^{2}}-\frac{h_{1}^{2}}{4096 \pi^{2}}$,

$\mu^{2} \frac{\mathrm{d}}{\mathrm{d} \mu^{2}}\left(3 L_{7}^{\mathrm{r}}+L_{8}^{\mathrm{r}}\right)=-\frac{5}{1536 \pi^{2}}$,

$\mu^{2} \frac{\mathrm{d}}{\mathrm{d} \mu^{2}}\left(2 L_{8}^{\mathrm{r}}-L_{5}^{\mathrm{r}}\right)=+\frac{1}{192 \pi^{2}}-\frac{3 h_{1}^{2}}{4096 \pi^{2}}$

is observed. We note that the large contribution proportional to $h_{2}^{2}$ in $2 L_{8}^{\mathrm{r}}-L_{5}^{\mathrm{r}}$ is reduced by a factor of $2 / 5$ as compared to the original expression (44). Suppose we have determined our low-energy parameters $L_{i}^{\mathrm{r}}$ from some data set. How would we confront them with the conventional low-energy parameters of Gasser and Leutwyler, which we denote here by $\bar{L}_{i}$ ? The required relations are provided with

$2 \bar{L}_{6}-\bar{L}_{4}=2 L_{6}^{\mathrm{r}}-L_{4}^{\mathrm{r}}-\frac{h_{1}^{2}}{4096 \pi^{2}} \log \frac{M_{\chi}^{2}}{\mu^{2}}$,

$3 \bar{L}_{7}+\bar{L}_{8}=3 L_{7}^{\mathrm{r}}+L_{8}^{\mathrm{r}}$,

$2 \bar{L}_{8}-\bar{L}_{5}=2 L_{8}^{\mathrm{r}}-L_{5}^{\mathrm{r}}-\frac{h_{1}^{2}}{8192 \pi^{2}}\left(-7+6 \log \frac{M_{\chi}^{2}}{\mu^{2}}\right)+\frac{h_{2}^{2}}{256 \pi^{2}}$.

We affirm that the scale dependence of the parameters, $\bar{L}_{i}$, resembles the one of the conventional $\chi \mathrm{PT}$ approach without dynamical vector mesons, i.e., the formulas in (43) taken at $h_{i}=0$.

It is instructive to compare our result to the well established one-loop expression of $\chi \mathrm{PT}$ in the absence of dynamical vector mesons. The corresponding expressions for the pion, kaon, and eta meson masses can readily be recognized in (38) and (39). We illustrate the role of the dynamical vector mesons in the ratio $\Pi_{P} / m_{P}^{2}$ as a function of $M_{\chi}$ at $\mu=M_{\chi}$. For this purpose we determine the product of $B_{0}^{\mathrm{r}}$ and the quark masses from the physical pion and kaon masses

$$
2 B_{0}^{\mathrm{r}} m \simeq m_{\pi}^{2}, \quad m_{s} \simeq 27.3 m,
$$



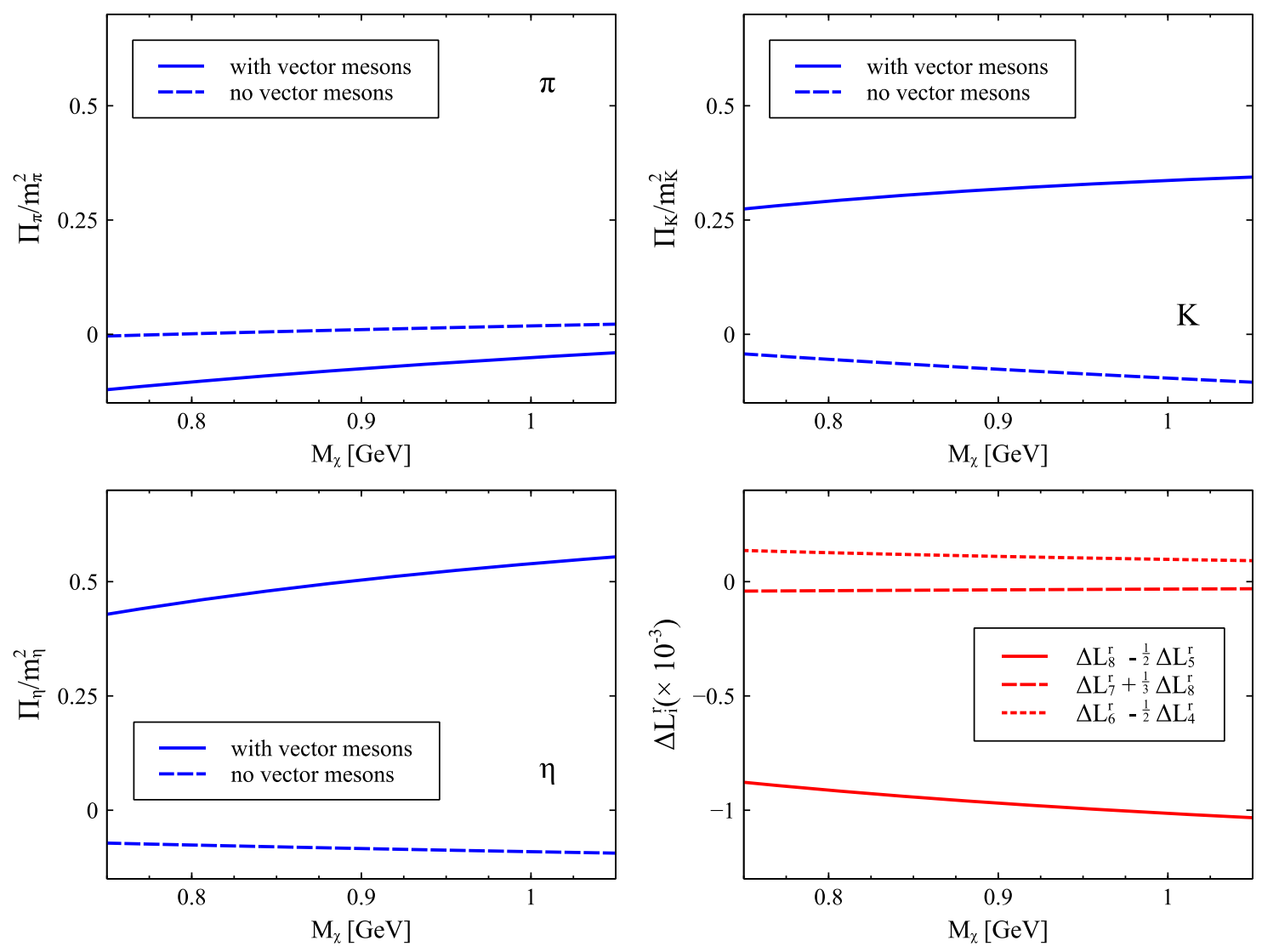

FIG. 5. The pseudoscalar meson polarizations $\Pi_{P} / m_{P}^{2}$ are plotted as a function of $M_{\chi}$, at $\mu=M_{\chi}$. For the meson masses inside the loop functions leading order expressions are used as described in the text with $\epsilon_{\omega}=\epsilon_{\phi}=0$ and $L_{i}^{\mathrm{r}}=0$. While the solid lines include the effect of vector meson loop contributions, the dashed lines leave the latter contributions out. In the last plot specific $\Delta L_{i}^{\mathrm{r}}$ are shown as functions of $M_{\chi}$. The $L_{i}^{\mathrm{r}}=\Delta L_{i}^{\mathrm{r}}$ are determined such that their effect would move the solid lines back on top of the dashed lines.

in terms of the GOR relation for the pion mass and the latest quark-mass ratio from the PDG [62]. Since we are after the typical size of loop effects, the contributions from the renormalized tree-level parameters are switched off with $L_{i}^{\mathrm{r}}=0$. As for our vector meson mass study we consider two cases both using $f_{\mathrm{r}}=90 \mathrm{MeV}$. In Fig. 5 we show the ratios $\Pi_{P} / m_{P}^{2}$ as determined from (39) and (40) with $M_{V, R} \rightarrow M_{\chi}$ and the kaon and eta meson masses approximated by the GOR relations, i.e., $m_{K}^{2}=B_{0}^{\mathrm{r}}\left(m+m_{s}\right)$ and $m_{\eta}^{2}=\frac{1}{3}\left(4 m_{K}^{2}-m_{\pi}^{2}\right)$. Figure 6 shows the same ratios evaluated with physical values for the masses of the pion, kaon, and eta mesons as well as all vector mesons. In both figures the subtraction rules (50) are imposed. Two lines are shown for the pion, kaon, and eta meson ratios always. While the solid lines show the effect including the contributions of the vector mesons, the dashed lines follow with $h_{1}=h_{2}=$ $h_{3}=0$ strictly for which there are no contributions from vector mesons.

In all cases we find a significant effect from the vector meson loop contributions. It is pointed out that such effects cannot be simply absorbed into the low-energy constants $L_{i}^{\mathrm{r}}$ as was worked out with (52). At the particular choice
$\mu=M_{\chi}$ the vector meson loop contributions renormalize exclusively the particular combination

$$
2 \bar{L}_{8}-\bar{L}_{5}=2 L_{8}^{\mathrm{r}}-L_{5}^{\mathrm{r}}+\underbrace{\frac{7 h_{1}^{2}}{8192 \pi^{2}}+\frac{h_{2}^{2}}{256 \pi^{2}}}_{\simeq 3.2 \times 10^{-3}},
$$

for which we provide its numerical estimate. With this one may have expected $\Delta L_{7}^{\mathrm{r}}+\Delta L_{8}^{\mathrm{r}} / 3=\Delta L_{6}^{\mathrm{r}}-\Delta L_{4}^{\mathrm{r}} / 2=0$ and $\Delta L_{8}^{\mathrm{r}}-\Delta L_{5}^{\mathrm{r}} / 2 \simeq-1.6$ in Fig. 5 or Fig. 6 . The latter values are far away from the results presented in the figures. We conclude that there are significant nonlinear structures from the vector meson loops that must not be expanded in the quark masses as suggested by conventional $\chi \mathrm{PT}$.

\section{DECAY CONSTANTS OF THE GOLDSTONE BOSONS}

We close this work with a study of the one-loop contributions to the decay constants $f_{P}$ of Goldstone bosons of type $P$. According to [51] the conventional approach leads to the following expressions: 

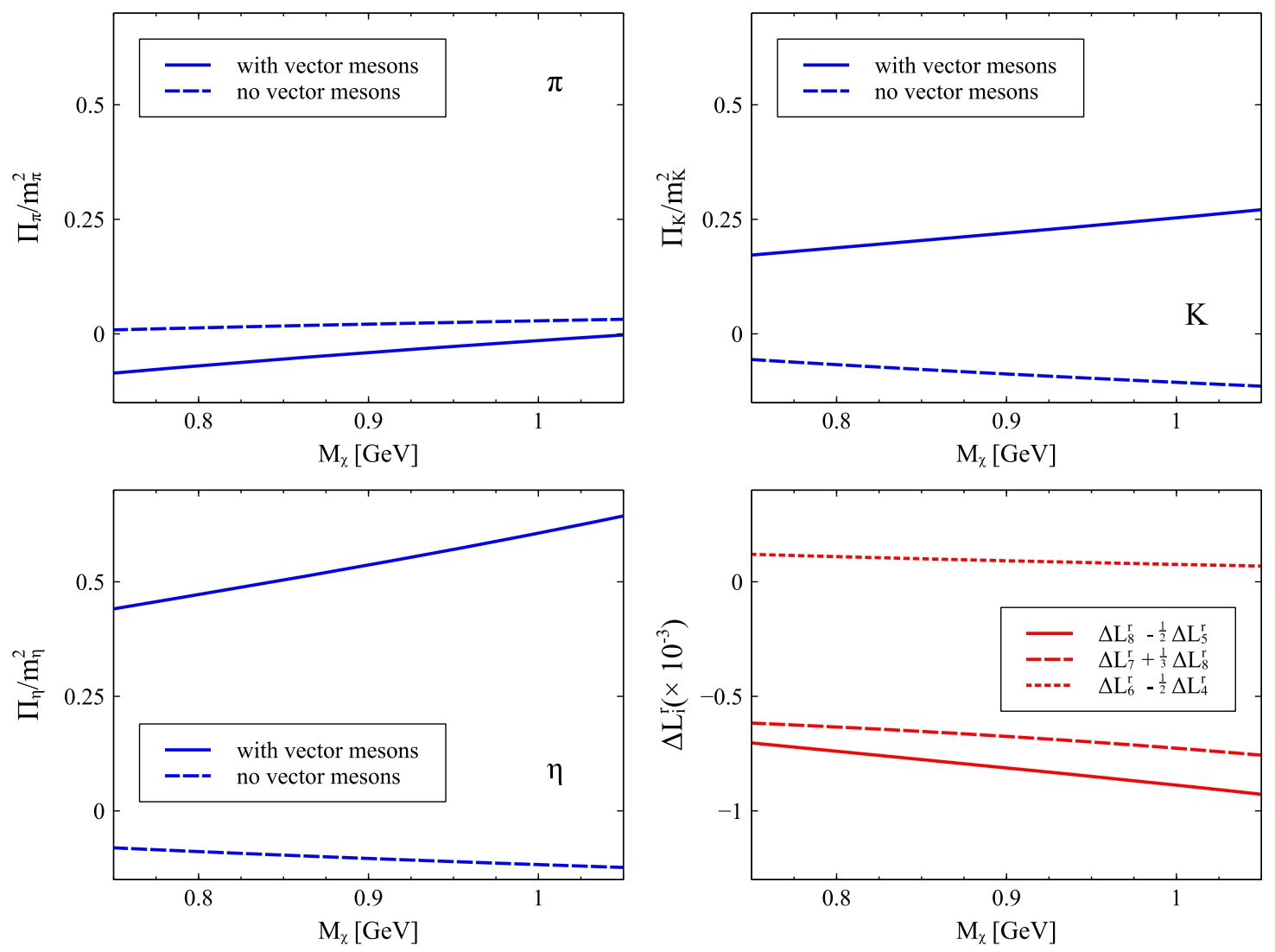

FIG. 6. The pseudoscalar meson polarizations $\Pi_{P} / m_{P}^{2}$ are plotted as a function of $M_{\chi}$, at $\mu=M_{\chi}=M_{r}$ and $L_{i}^{\mathrm{r}}=0$. For the meson masses inside the loop functions physical values are assumed. For the mixing angles we use $\epsilon_{\omega}=0.45$ and $\epsilon_{\phi}=0.058$. While the solid lines include the effect of vector meson loop contributions, the dashed lines leave the latter contributions out. In the last plot specific $\Delta L_{i}^{\mathrm{r}}$ are shown as functions of $M_{\chi}$. The $L_{i}^{\mathrm{r}}=\Delta L_{i}^{\mathrm{r}}$ are determined such that their effect would move the solid lines back on top of the dashed lines.

$$
\begin{aligned}
& f_{\pi}^{\chi-\mathrm{PT}}=f-\frac{1}{f} \bar{I}_{\pi}-\frac{1}{2 f} \bar{I}_{K}+\underbrace{\frac{8 B_{0} m}{f}}_{\rightarrow 4 m_{\pi}^{2} / f} L_{5}+\underbrace{\frac{8 B_{0}\left(2 m+m_{s}\right)}{f}}_{\rightarrow 4\left(2 m_{K}^{2}+m_{\pi}^{2}\right) / f} L_{4}, \\
& f_{K}^{\chi-\mathrm{PT}}=f-\frac{3}{8 f} \bar{I}_{\pi}-\frac{3}{4 f} \bar{I}_{K}-\frac{3}{8 f} \bar{I}_{\eta}+\underbrace{\frac{4 B_{0}\left(m+m_{s}\right)}{f}}_{\rightarrow 4 m_{K}^{2} / f} L_{5}+\underbrace{\frac{8 B_{0}\left(2 m+m_{s}\right)}{f}}_{\rightarrow 6\left(m_{\eta}^{2}+m_{\pi}^{2}\right) / f} L_{4}, \\
& f_{\eta}^{\chi-\mathrm{PT}}=f-\frac{3}{2 f} \bar{I}_{K}+\underbrace{\frac{8 B_{0}\left(m+2 m_{s}\right)}{3 f}}_{4 m_{\eta}^{2} / f} L_{5}+\underbrace{\frac{8 B_{0}\left(2 m+m_{s}\right)}{f}}_{\rightarrow 12\left(2 m_{K}^{2}-m_{\eta}^{2}\right) / f} L_{4},
\end{aligned}
$$

with the tadpole integrals as given in (39). Before providing the additional contributions that arise from the presence of dynamical vector mesons we further illuminate our scheme formulated in terms of physical masses. Within the conventional $\chi \mathrm{PT}$ approach the pion, kaon, and $\eta$ meson masses that enter the tadpole integrals $\bar{I}_{Q}$ in (56) need to be approximated by the leading order expressions, i.e., $m_{\pi}^{2} \rightarrow 2 m B_{0}$, etc. If done so, the expressions for the decay constants will not depend on the renormalization scale $\mu$. However, it would clearly be instrumental if we could keep the physical masses inside the loops without giving up on the rigor of conventional $\chi \mathrm{PT}$. An initial attempt where one simply kept the tadpole terms with physical masses suffers from an uncontrolled scale dependence of the resulting 
expressions for the decay constants. Is it possible to identify the higher order terms that would again lead to renormalization scale invariance? Such terms should be determined by a renormalization group equation. Indeed, it is possible to construct such terms unambiguously. All that is needed is to reinterpret the quark-mass terms in (56) by suitable combinations of the pion, kaon, and $\eta$ meson masses as indicated by the replacement rules in (56). We assure the reader that with the latter the physical masses in the tadpole terms can be used without being punished by a scale dependence in the decay constants.

We turn now to the contributions from the dynamical vector meson degree of freedom. As for the vector meson masses such terms will renormalize the chiral limit value of $f_{P}$ away from the parameter $f$. Altogether, for the decay constants we find

$f_{P \in[8]}=f_{P}^{\chi-\mathrm{PT}}+\frac{3}{4 f} \sum_{V \in[9]} G_{P V}^{(T)} \bar{I}_{V}+\left(\frac{f}{2} \frac{\partial}{\partial m_{P}^{2}}-\frac{f}{m_{P}^{2}}\right) \Pi_{P}^{\text {bubble }}$

where we point to the close correspondence of (40) and (57). In particular, all coefficient $G_{P V}^{(T)}$ have been introduced before in (40) and are listed in Table IV.

As in the previous section we first determine the scale dependence of the relevant low-energy parameters in strict perturbation theory. For this purpose we need to decompose $f$ into its power counting moments with

$$
f=\sum_{n=0}^{\infty} M^{2 n} f^{(2 n)}
$$

While the leading order moment $f^{(0)}$ remains scale invariant, the second order moment $f^{(2)}$ does depend on the renormalization scale. Altogether we derive

$$
\begin{aligned}
\mu^{2} \frac{\mathrm{d}}{\mathrm{d} \mu^{2}} f^{(2)}= & +\frac{9\left(4 g_{1}+4 g_{2}+2 g_{3}-g_{5}\right)}{128 \pi^{2} f^{(0)}}+\frac{18 h_{1}^{2}}{1024 \pi^{2} f^{(0)}} \\
& +\frac{63 h_{2}^{2}}{512 \pi^{2} f^{(0)}} \\
\mu^{2} \frac{\mathrm{d}}{\mathrm{d} \mu^{2}} L_{4}= & -\frac{1}{256 \pi^{2}}+\frac{3 h_{1}^{2}}{4096 \pi^{2}}, \\
\mu^{2} \frac{\mathrm{d}}{\mathrm{d} \mu^{2}} L_{5}= & -\frac{3}{256 \pi^{2}}+\frac{9 h_{1}^{2}}{4096 \pi^{2}} .
\end{aligned}
$$

It remains to identify the renormalized low-energy parameters. Again they follow upon a quark-mass expansion of the loop function that involves the dynamical vector mesons. We introduce

$$
\begin{aligned}
f^{\text {ren }}= & f+\frac{9\left(4 g_{1}+4 g_{2}+2 g_{3}-g_{5}\right)}{128 \pi^{2} f} M^{2} \log \frac{M^{2}}{\mu^{2}} \\
& +\frac{3 h_{1}^{2} M^{2}}{1024 \pi^{2} f}\left(1+6 \log \frac{M^{2}}{\mu^{2}}\right) \\
& +\frac{3 h_{2}^{2} M^{2}}{512 \pi^{2} f}\left(16+21 \log \frac{M^{2}}{\mu^{2}}\right), \\
L_{4}^{\text {ren }}= & L_{4}+\frac{h_{1}^{2}}{8192 \pi^{2}}\left(1+6 \log \frac{M^{2}}{\mu^{2}}\right), \\
L_{5}^{\text {ren }}= & L_{5}+\frac{3 h_{1}^{2}}{8192 \pi^{2}}\left(1+6 \log \frac{M^{2}}{\mu^{2}}\right) .
\end{aligned}
$$

As we observed for the low-energy parameters $B_{0}$ and $2 L_{7}-L_{5}$, there is a significant contribution from the $h_{2}$ coupling constant in the renormalized expression for the low-energy parameter $f$. The latter is about a factor of 20 larger than the corresponding term induced by the $h_{1}$ coupling constant. The results for the renormalized $L_{4}$ and $L_{5}$ parameters are in line with expressions given previously in the literature $[44,45]$. There is no contribution from $h_{2}$ in this case. We iterate that it is mandatory to resum all terms proportional to $\left(M / \Lambda_{\chi}\right)^{n}$. The expressions (60) as they stand are not significant.

Again we impose the subtraction rules (50) in (57) that are expected to generate the desired summation effects (47) we are after. We assure the reader that as an immediate consequence of (50) the low-energy parameter $f_{\mathrm{r}}$ is not renormalized by loop effects. This implies

$$
\left[f_{P}-f_{P}^{\chi-P T}\right]_{\text {renormalized }} \sim \frac{B_{0}^{\mathrm{r}} m_{\text {quark }}}{\left(4 \pi f_{\mathrm{r}}\right)^{2}} f_{\mathrm{r}}
$$

in the chiral domain with the quark masses approaching the chiral limit. We note that there is no explicit dependence left on any of the unknown low-energy parameters $g_{n}$. Moreover, we can safely use physical masses in all loop expressions. Scale invariant results arise for the decay constants if and only if the replacement rules indicated already in (56) are imposed. It remains to identify the lowenergy parameters $\bar{L}_{4}$ and $\bar{L}_{5}$ for which we obtain

$$
\bar{L}_{4}=L_{4}^{\mathrm{r}}+\frac{h_{1}^{2}}{4096 \pi^{2}} \log \frac{M_{\chi}^{2}}{\mu^{2}}, \quad \bar{L}_{5}=L_{5}^{\mathrm{r}}+\frac{3 h_{1}^{2}}{4096 \pi^{2}} \log \frac{M_{\chi}^{2}}{\mu^{2}} .
$$

We are now prepared to illustrate the role of vector meson loop contributions in the decay constants of the Goldstone bosons. In Figs. 7 and 8 we plot the normalized ratio $f_{P} / f_{\mathrm{r}}-1$ as a function of $M_{\chi}$ at $\mu=M_{\chi}$ together with

$$
f_{\mathrm{r}}=90 \mathrm{MeV}, \quad L_{4}^{\mathrm{r}}=L_{5}^{\mathrm{r}}=0
$$



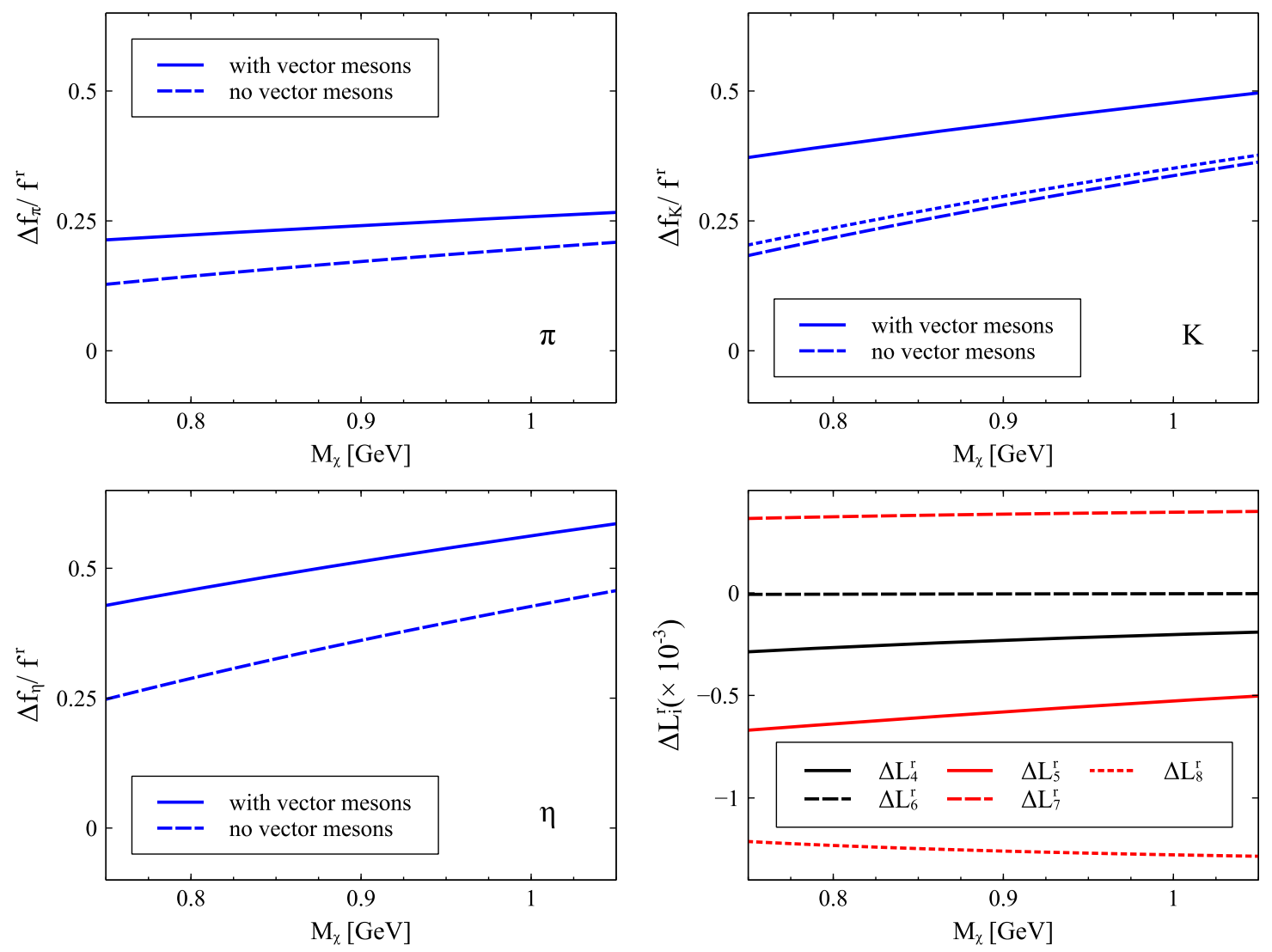

FIG. 7. The ratios $\Delta f_{P} / f_{\mathrm{r}}$ are plotted as a function of $M_{\chi}$ at $\mu=M_{\chi}=M_{r}$ and $L_{i}^{\mathrm{r}}=0$. For the meson masses inside the loop functions leading order expressions are used as in Fig. 5. While the solid lines include the effect of vector meson loop contributions, the dashed lines leave the latter contributions out. In the last plot specific $\Delta L_{i}^{\mathrm{r}}$ are shown as functions of $M_{\chi}$. The $L_{i}^{\mathrm{r}}=\Delta L_{i}^{\mathrm{r}}$ are determined such that their effect would move the solid lines in the pion and eta meson box of Fig. 5 and in this figure back on top of the dashed lines. The dotted line in the kaon box shows the effect of the $\Delta L_{i}^{\mathrm{r}}$ on the kaon decay constant.

As in Figs. 5 and 6 we show the results of using approximated and physical meson masses, respectively. Two lines are shown for the normalized ratios of the pion, kaon, and eta meson decay constants. While the solid lines show the effect including the contributions of the vector mesons, the dashed lines follow with $h_{1}=h_{2}=h_{3}=0$ strictly for which there are no contributions from vector mesons. The low-energy parameters $L_{4}^{\mathrm{r}}=\Delta L_{4}^{\mathrm{r}}$ and $L_{5}^{\mathrm{r}}=\Delta L_{5}^{\mathrm{r}}$ can be adjusted to cancel the effect of the vector meson loop contributions to the pion and eta meson decay constants. Given the scenario (54) with $f_{\mathrm{r}}=90 \mathrm{MeV}$ we determine the low-energy constants as a function of $M_{\chi}$. We observe again that the use of physical meson masses in the loop functions play an important role.

We recall that if the vector meson loop contributions would be approximated well by conventional $\chi \mathrm{PT}$ structures at order $Q^{4}$, we would have obtained the specific values

$$
\Delta L_{4}^{\mathrm{r}}=\Delta L_{5}^{\mathrm{r}}=\Delta L_{6}^{\mathrm{r}}=0, \quad \Delta L_{8}^{\mathrm{r}} \simeq-1.6, \quad \Delta L_{7}^{\mathrm{r}} \simeq 0.5 .
$$

As is clearly shown by Figs. 7 and 8 we are far from such a situation. Thus we conclude it is important to consider dynamical vector meson degrees of freedom in a chiral extrapolation attempt of meson masses in QCD.

\section{SETS OF LOW-ENERGY CONSTANTS}

It is useful to summarize some scenarios for the renormalized low-energy parameters as they were introduced and discussed in our work. Though, given the physical meson masses and decay constants only, it is not possible to determine any of the low-energy parameters, we can nevertheless illustrate their typical size. We do so by making ad hoc choices for some of the parameters and then determine the values of the remaining parameters by the request that the physical masses and decay constants $f_{\pi}$ and $f_{K}$ are reproduced. This is the rationality behind most of the figures shown in our work. In Table VI such choices are detailed.

It is emphasized that significant results for the parameters $f_{\mathrm{r}}$ and $M_{r}$ in Table VI can be expected only after 

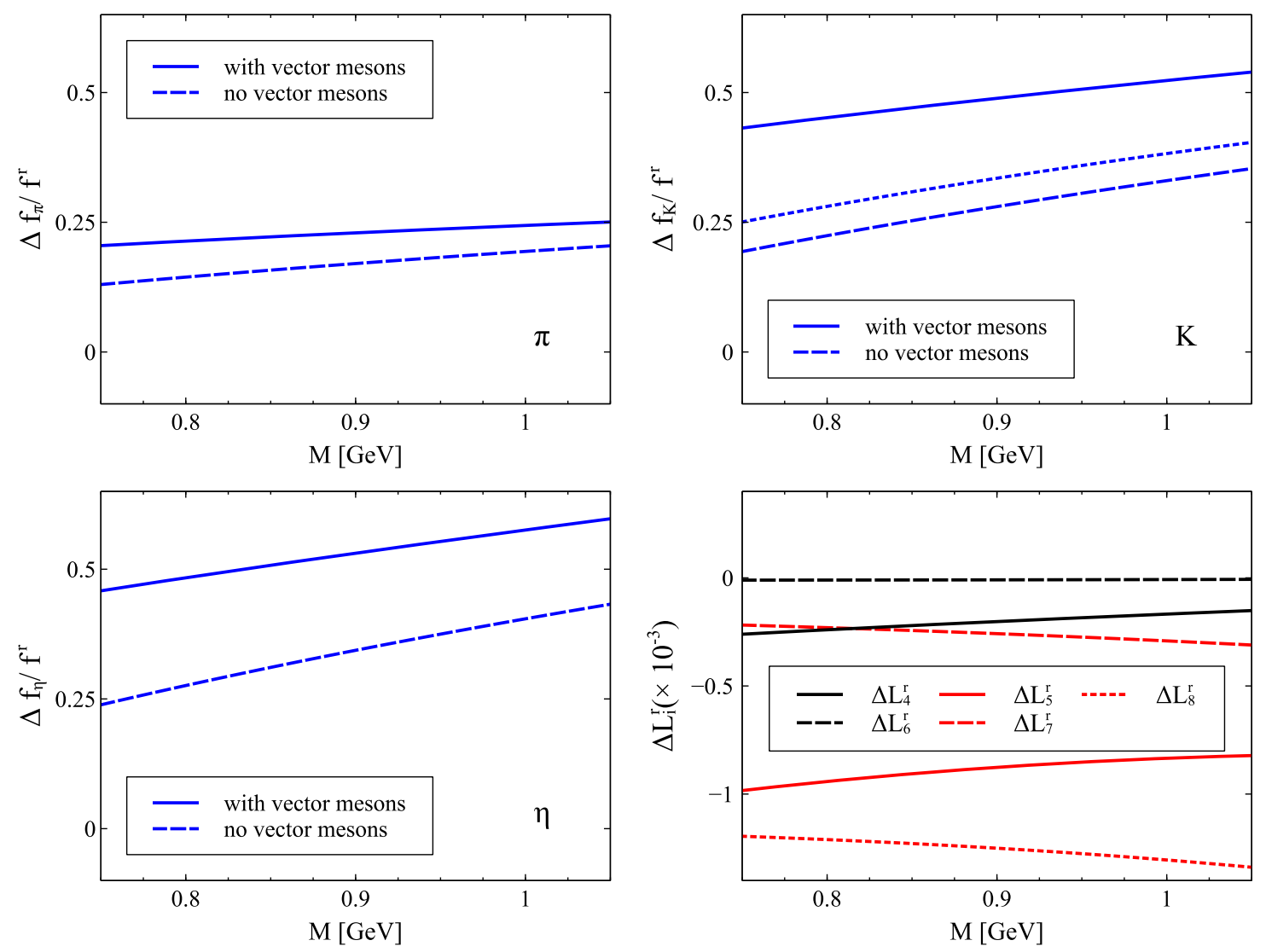

FIG. 8. The ratios $\Delta f_{P} / f_{\mathrm{r}}$ are plotted as a function of $M_{\chi}$ at $\mu=M_{\chi}=M_{r}$ and $L_{i}^{\mathrm{r}}=0$. For the meson masses inside the loop functions physical values are used as in Fig. 6. While the solid lines include the effect of vector meson loop contributions, the dashed lines leave the latter contributions out. In the last plot specific $\Delta L_{i}^{\mathrm{r}}$ are shown as functions of $M_{\chi}$. The $L_{i}^{\mathrm{r}}=\Delta L_{i}^{\mathrm{r}}$ are determined such that their effect would move the solid lines in the pion and eta meson boxes of Fig. 6 and this figure back on top of the dashed lines. The dotted line in the kaon box shows the effect of the $\Delta L_{i}^{\mathrm{r}}$ on the kaon decay constant.

their values are adjusted to QCD lattice simulation data on the meson masses and their decay constants. Since such data are available at unphysical choices for the quark masses, additional constraints arise that should be used to establish the values for such low-energy parameters.

Given the low-energy parameters in Table VI we can infer values for the remaining low-energy parameters, the only exceptions being the low-energy parameters $c_{2}^{\mathrm{r}}$ and $g_{n}$. In Table VII we display the values implied by the physical meson masses and decay constants at various values for $c_{2}^{\mathrm{r}}$. We emphasize that most low-energy constants depend quite strongly on the particular choices for $c_{2}^{\mathrm{r}}$ but also $f_{\mathrm{r}}, M_{r}$. Note that in the infinite volume limit none of the parameters $g_{n}$ have an impact on

TABLE VI. Ad hoc choice for some renormalized low-energy constants.

\begin{tabular}{lcccccc}
\hline \hline$f_{\mathrm{r}}$ & $M_{r}$ & $h_{1}$ & $h_{2}$ & $h_{3}$ & $\left|\epsilon_{\phi}\right|$ & $\mu$ \\
\hline $0.09 \mathrm{GeV}$ & $0.8 \mathrm{GeV}$ & 2.5 & $2.33 / \cos \epsilon_{\omega}$ & 0.049 & 0.058 & $0.8 \mathrm{GeV}$ \\
\hline \hline
\end{tabular}

any meson mass or decay constant. Thus, there is no way to provide an estimate for such parameters at this stage.

TABLE VII. Low-energy parameters as implied by ad hoc values for $c_{2}^{r}$ and the low-energy constants of Table VI.

\begin{tabular}{lrrrrrr}
\hline \hline$c_{2}^{\mathrm{r}}$ & -1 & 0 & 1 & -1 & 0 & 1 \\
\hline$e_{2}^{\mathrm{r}}$ & -0.175 & -0.223 & -0.289 & -0.297 & -0.384 & -0.488 \\
$\epsilon_{\phi}$ & 0.058 & 0.058 & 0.058 & -0.058 & -0.058 & -0.058 \\
$\epsilon_{\omega}$ & 0.338 & 0.427 & 0.524 & 0.472 & 0.562 & 0.647 \\
$b_{1}^{\mathrm{r}}$ & 0.908 & 0.025 & -0.888 & 0.545 & -0.394 & -1.358 \\
$b_{2}^{\mathrm{r}}$ & 0.433 & 0.512 & 0.614 & 0.519 & 0.628 & 0.757 \\
$b_{3}^{\mathrm{r}}$ & 0.893 & 1.124 & 1.437 & 1.222 & 1.581 & 2.010 \\
$c_{1}^{\mathrm{r}}$ & -0.212 & 0.095 & 0.396 & 0.354 & 0.702 & 1.051 \\
$L_{4}^{\mathrm{r}} \times 10^{3}$ & -0.743 & -0.740 & -0.737 & -0.737 & -0.734 & -0.732 \\
$L_{5}^{\mathrm{r}} \times 10^{3}$ & -0.376 & -0.447 & -0.530 & -0.534 & -0.612 & -0.691 \\
$L_{6}^{\mathrm{r}} \times 10^{3}$ & -0.273 & -0.272 & -0.269 & -0.273 & -0.271 & -0.268 \\
$L_{7}^{\mathrm{r}} \times 10^{3}$ & -0.358 & -0.431 & -0.524 & -0.581 & -0.681 & -0.807 \\
$L_{8}^{\mathrm{r}} \times 10^{3}$ & -0.941 & -1.003 & -1.109 & -0.982 & -1.093 & -1.244 \\
\hline \hline
\end{tabular}




\section{SUMMARY AND OUTLOOK}

In this work we scrutinized the hadrogenesis Lagrangian, a chiral $S U(3)$ interaction with explicit vector meson degrees of freedom in the tensor field representation. Based on the leading order interaction the one-loop contributions to the vector meson masses were computed in application of dimensional counting rules. We found that six parameters from the original version of the Lagrangian need to be moved to a higher order to arrive at a consistent renormalization program. This is an important finding since this further increases the predictive power of the hadrogenesis Lagrangian.

The subtle interplay of the hadrogenesis mass gap scale $\Lambda_{\mathrm{HG}}$ and the chiral symmetry breaking scale $\Lambda_{\chi}$ was discussed. The dimensional counting rules rely on the assumption $M / \Lambda_{\mathrm{HG}}$, with $M$ the vector meson mass in the chiral and large- $N_{c}$ limit of QCD. At sufficiently large $N_{c}$ with $\Lambda_{\chi} \geq \Lambda_{\mathrm{HG}}$ the hadrogenesis Lagrangian can be applied in perturbation theory. For the physical choice $N_{c}=3$ with $\Lambda_{\chi}<\Lambda_{\mathrm{HG}}$ a partial summation of all terms proportional to $M / \Lambda_{\chi} \sim 1$ is required to arrive at significant results. It was suggested that this can be achieved by a suitable renormalization scheme. First, numerical estimates for the size of the one-loop corrections for the vector meson masses were provided given such a framework. The results are in line with the expectation of the dimensional counting rules.

The work was supplemented by computations of the oneloop corrections of the masses and decay constants of the Goldstone bosons. The size of the loop contributions for vector meson degrees of freedom was illustrated by a series of figures, which suggest good convergence properties of the effective field theory.

Further steps are required to consolidate our findings on the crucial importance of dynamical vector meson degrees of freedom. The result obtained in this work can be used for an attempt to describe the quark-mass dependence of unquenched QCD lattice simulation data on the vector mesons as well as on the masses and decay constants of the Goldstone bosons. Such data are expected to determine some of the so far unknown low-energy constants of the hadrogenesis Lagrangian and constitute a quantitative challenge of the framework.

Once the framework would pass such a stringent test we would deem it justified to apply the hadrogenesis Lagrangian to meson phenomenology of chiral dynamics in QCD below the hadrogenesis scale. This may require one to work out additional constraints on the form of the hadrogenesis Lagrangian that are expected from a one-loop study of the vector meson scattering amplitudes. It is also left to investigate the axial anomaly sector with its $\eta^{\prime}$ meson, which was not considered yet in the current study.

\section{ACKNOWLEDGMENTS}

Financial support from the Thailand Research Fund through the Royal Golden Jubilee Ph.D. Program (Grant No. PHD/0227/2553) to R. B. is acknowledged. We thank C. Kobdaj for useful discussions.

\section{APPENDIX}

The chiral expansions of scalar bubbles read

$$
\begin{aligned}
& I_{Q P}=\frac{1}{16 \pi^{2}}\left[1-\log \frac{M^{2}}{\mu^{2}}+\frac{m_{P}^{2}}{M^{2}}\left(1-\log \frac{m_{P}^{2}}{M^{2}}\right)+\frac{m_{Q}^{2}}{M^{2}}\left(1-\log \frac{m_{Q}^{2}}{M^{2}}\right)-\frac{m_{Q}^{4}}{2 M^{4}}-\frac{m_{P}^{4}}{2 M^{4}}-\frac{m_{Q}^{2} m_{P}^{2}}{M^{4}}\left(\log \frac{m_{P}^{2}}{\bar{M}^{2}}+\log \frac{m_{Q}^{2}}{M^{2}}\right)+\cdots\right], \\
& I_{Q R}=\frac{1}{16 \pi^{2}}\left[1-\log \frac{M^{2}}{\mu^{2}}-\frac{m_{Q} \pi}{M}+\frac{m_{Q}^{2}}{2 M^{2}}\left(2-\log \frac{m_{Q}^{2}}{M^{2}}\right)+\frac{m_{Q}^{3}}{8 M^{3}}-\frac{m_{Q}^{4}}{12 M^{4}}+\cdots\right], \\
& I_{R T}=\frac{1}{16 \pi^{2}}\left[1-\frac{\pi}{\sqrt{3}}-\log \left(\frac{M^{2}}{\mu^{2}}\right)+\cdots\right],
\end{aligned}
$$

where the vector meson masses are assigned to be their chiral limit $M_{V}=M_{R}=M_{T}=M$.

The strict chiral expansion of the scalar bubbles,

$$
\begin{gathered}
\bar{I}_{Q V}=\frac{1}{16 \pi^{2}}\left[\frac{m_{P}^{2}}{2 M^{2}}+\frac{m_{Q}^{2}}{M^{2}} \log \left(\frac{m_{Q}^{2}}{M^{2}}\right)+\frac{m_{P}^{4}+9 m_{P}^{2} m_{Q}^{2}}{6 M^{4}}+\frac{m_{Q}^{2}}{M^{4}}\left(m_{P}^{2}+m_{Q}^{2}\right) \log \left(\frac{m_{Q}^{2}}{M^{2}}\right)+\cdots\right], \\
\bar{I}_{V R}=\underbrace{-\frac{1}{16 \pi^{2}}}_{\text {from the chiral limit of } I_{V R}(0), \text { so } \Delta I_{V R} \text { is free from this term }}+\frac{1}{16 \pi^{2}}\left(\frac{m_{P}^{2}}{6 M^{2}}+\frac{m_{P}^{4}}{60 M^{4}}\right)+\cdots .
\end{gathered}
$$

In the above expansion, the vector meson masses are evaluated at the chiral limit $M_{V}=M_{R}=M$. 
[1] J. J. Sakurai, Currents and Mesons (University of Chicago Press, Chicago, 1969).

[2] H. Georgi, Phys. Rev. Lett. 63, 1917 (1989).

[3] H. Georgi, Nucl. Phys. B331, 311 (1990).

[4] U. G. Meißner, Phys. Rep. 161, 213 (1988).

[5] J. Bijnens and E. Pallante, Mod. Phys. Lett. A 11, 1069 (1996).

[6] M. C. Birse, Z. Phys. A 355, 231 (1996).

[7] J. Bijnens, G. Colangelo, and G. Ecker, J. High Energy Phys. 02 (1999) 020.

[8] F. Klingl, N. Kaiser, and W. Weise, Z. Phys. A 356, 193 (1996).

[9] M. F. M. Lutz, G. Wolf, and B. Friman, Nucl. Phys. A706, 431 (2002); A765, 495(E) (2006).

[10] D. Djukanovic, M. R. Schindler, J. Gegelia, G. Japaridze, and S. Scherer, Phys. Rev. Lett. 93, 122002 (2004).

[11] P. C. Bruns and U.-G. Meißner, Eur. Phys. J. C 40, 97 (2005).

[12] D. Djukanovic, J. Gegelia, A. Keller, and S. Scherer, Phys. Lett. B 680, 235 (2009).

[13] M. F. M. Lutz and S. Leupold, Nucl. Phys. A813, 96 (2008).

[14] C. Terschlüsen, S. Leupold, and M. F. M. Lutz, Eur. Phys. J. A 48, 190 (2012).

[15] M. Bando, T. Kugo, and K. Yamawaki, Phys. Rep. 164, 217 (1988).

[16] C. Vafa and E. Witten, Nucl. Phys. B234, 173 (1984).

[17] M. Tanabashi, Phys. Lett. B 316, 534 (1993).

[18] M. Harada and K. Yamawaki, Phys. Rep. 381, 1 (2003).

[19] P. Ko and S. Rudaz, Phys. Rev. D 50, 6877 (1994).

[20] G. W. Carter, P. J. Ellis, and S. Rudaz, Nucl. Phys. A603, 367 (1996); A608, 514(E) (1996).

[21] M. Urban, M. Buballa, and J. Wambach, Nucl. Phys. A697, 338 (2002).

[22] D. Parganlija, P. Kovacs, G. Wolf, F. Giacosa, and D. H. Rischke, Phys. Rev. D 87, 014011 (2013).

[23] E. E. Jenkins, A. V. Manohar, and M. B. Wise, Phys. Rev. Lett. 75, 2272 (1995).

[24] J. Bijnens and P. Gosdzinsky, Phys. Lett. B 388, 203 (1996).

[25] J. Bijnens, P. Gosdzinsky, and P. Talavera, Nucl. Phys. B501, 495 (1997).

[26] J. Bijnens, P. Gosdzinsky, and P. Talavera, Phys. Lett. B 429, 111 (1998).

[27] D. Djukanovic, J. Gegelia, and S. Scherer, Int. J. Mod. Phys. A 25, 3603 (2010).

[28] P. C. Bruns, L. Greil, and A. Schäfer, Phys. Rev. D 88, 114503 (2013).

[29] T. Fuchs, M. R. Schindler, J. Gegelia, and S. Scherer, Phys. Lett. B 575, 11 (2003).

[30] K. Kampf, J. Novotny, and J. Trnka, Phys. Rev. D 81, 116004 (2010).

[31] A. Pich, I. Rosell, and J. J. Sanz-Cillero, J. High Energy Phys. 07 (2008) 014.

[32] Z.-H. Guo and J. J. Sanz-Cillero, Phys. Rev. D 89, 094024 (2014).

[33] M. F. M. Lutz and E. E. Kolomeitsev, Nucl. Phys. A700, 193 (2002).

[34] M. F. M. Lutz and E. E. Kolomeitsev, Found. Phys. 31, 1671 (2001).
[35] M. F. M. Lutz and E. E. Kolomeitsev, Nucl. Phys. A730, 392 (2004).

[36] E. E. Kolomeitsev and M. F. M. Lutz, Phys. Lett. B 582, 39 (2004).

[37] E. E. Kolomeitsev and M. F. M. Lutz, Phys. Lett. B 585, 243 (2004).

[38] M. F. M. Lutz and M. Soyeur, Nucl. Phys. A813, 14 (2008).

[39] E. van Beveren, T. A. Rijken, K. Metzger, C. Dullemond, G. Rupp, and J. E. Ribeiro, Z. Phys. C 30, 615 (1986).

[40] J. D. Weinstein and N. Isgur, Phys. Rev. D 41, 2236 (1990).

[41] L. Roca, E. Oset, and J. Singh, Phys. Rev. D 72, 014002 (2005).

[42] M. Wagner and S. Leupold, Phys. Rev. D 78, 053001 (2008).

[43] E. Witten, Nucl. Phys. B156, 269 (1979).

[44] C. Terschlüsen and S. Leupold, arXiv:1604.01682.

[45] C. Terschlüsen and S. Leupold, Phys. Rev. D 94, 014021 (2016).

[46] A. Krause, Helv. Phys. Acta 63, 3 (1990).

[47] G. Ecker, J. Gasser, A. Pich, and E. de Rafael, Nucl. Phys. B321, 311 (1989).

[48] G. Ecker, J. Gasser, H. Leutwyler, A. Pich, and E. de Rafael, Phys. Lett. B 223, 425 (1989).

[49] P. Herrera-Siklody, J. I. Latorre, P. Pascual, and J. Taron, Nucl. Phys. B497, 345 (1997).

[50] R. Kaiser and H. Leutwyler, Eur. Phys. J. C 17, 623 (2000).

[51] J. Gasser and H. Leutwyler, Nucl. Phys. B250, 465 (1985).

[52] S. Okubo, Phys. Lett. 5, 165 (1963).

[53] A. Kucukarslan and U.-G. Meißner, Mod. Phys. Lett. A 21, 1423 (2006).

[54] Also the subleading parameters $c_{3}, c_{4}$, and $c_{7}$ in (12) do not do so. With

$$
\begin{aligned}
c_{6}+2 c_{8}+4 c_{9}+8 c_{10} & =0, \quad c_{6}+c_{8}+2 c_{9}+2 c_{10}=0, \\
2 c_{5}+3 c_{8}+8 c_{10} & =0,
\end{aligned}
$$

conditions are obtained that exclude any mixing effect.

[55] M. F. M. Lutz, R. Bavontaweepanya, C. Kobdaj, and K. Schwarz, Phys. Rev. D 90, 054505 (2014).

[56] Upon the replacement

$$
G_{\ldots}^{(V)} G_{\ldots}^{(V)} \rightarrow G_{\ldots}^{(\omega)} G_{\ldots}^{(\phi)}
$$

the bubble loop contribution to $\Pi_{\omega \phi}$ is readily constructed from (18).

[57] D. B. Leinweber, A. W. Thomas, K. Tsushima, and S. V. Wright, Phys. Rev. D 64, 094502 (2001).

[58] A. Semke and M. F. M. Lutz, Nucl. Phys. A778, 153 (2006).

[59] M. F. M. Lutz, Y. Heo, and X.-Y. Guo, Nucl. Phys. A977, 146 (2018).

[60] X.-Y. Guo, Y. Heo, and M. F. M. Lutz, Phys. Rev. D 98, 014510 (2018).

[61] M. F. M. Lutz, Nucl. Phys. 677, 241 (2000).

[62] C. Patrignani et al. (Particle Data Group Collaboration), Chin. Phys. C 40, 100001 (2016).

[63] J. Gasser and H. Leutwyler, Ann. Phys. (N.Y.) 158, 142 (1984). 